\title{
Enhanced Ehlers transformation and the Majumdar-Papapetrou-NUT spacetime
}

\author{
Marco Astorino \\ Istituto Nazionale di Fisica Nucleare (INFN), Sezione di Milano, \\ Via Celoria 16, Milano I-20133, Italy \\ E-mail: Marco.Astorino@Gmail.com
}

ABSTRACT: The transformation which adds (or removes) NUT charge when it is applied to electrovacuum, axisymmetric and stationary space-times is studied. After analysing the Ehlers and the Reina-Treves transformations we propose a new one, more precise in the presence of the Maxwell electromagnetic field. The enhanced Ehlers transformation proposed turns out to act as a gravitomagnetic duality, analogously to the electromagnetic duality, but for gravity: it rotates the mass charge into the gravomagnetic (or NUT) charge.

As an example the Kerr-Newman-NUT black hole is obtained with the help of this enhanced transformation.

Moreover a new analytical exact solution is built adding the NUT charge to a double charged black hole, at equilibrium. It describes the non-extremal generalisation of the Majumdar-Papapetrou-NUT solution. From the near-horizon analysis, its microscopic entropy, according to the Kerr/CFT correspondence, is found and the second law of black hole thermodynamics is discussed.

Keywords: Black Holes, Classical Theories of Gravity, Duality in Gauge Field Theories, Gauge-gravity correspondence

ARXIV EPRINT: 1906.08228 


\section{Contents}

1 Introduction 1

2 Review of the Ernst generating technique 2

3 Example: adding NUT to the Kerr-Newman black hole 5

4 Enhanced Ehlers transformations and gravitomagnetic duality 9

$5 \quad$ NUT generalisation of Majumdar-Papapetrou black holes 13

5.1 The non-extremal case: NUT generalisation of a RN black holes pair $\quad 14$

5.2 The extremal case: Majumdar-Papapetrou-NUT spacetime 16

5.3 Warm up: Reissner-Nordstrom-NUT/CFT correspondence 22

5.4 Near horizon geometry of the Majumdar-Papapetrou-(NUT) spacetime 24

5.5 Second law of thermodynamics 26

6 Summary, comments and conclusions 28

A Notation: differential operators in various coordinates 29

B Kerr-Newman-NUT solution from the enhanced Ehlers transformation 30

B.1 Multipole moments expansion for the Kerr-NUT spacetime 32

C NUT generalisation of the Majumdar-Papapetrou in $(\rho, z)$ coordinates 34

\section{Introduction}

Recent gravitational waves detection confirms the existence of binary black holes systems. Actually because of the enormous emission of gravitational radiation provided by the interaction of black hole pairs, this will be the most abundant and relevant source of gravitational waves data in the near future.

Numerical relativity is developing some powerful tools to study black hole coalescence and merging. However analytical and exact solutions to qualitatively study phenomena involving an ensemble of interacting black holes are scarce. The few known belong to the class of static solutions, most of all supported by extra matter, such as cosmic strings, to sustain the gravitational attraction between the two sources and avoid the gravitational collapse, see for example [1] or [2]. Actually, in the context of static metrics, it have been shown that, under certain reasonable regularity assumptions, the only multi black hole configuration is an extremal ensemble of charged sources [3, 25].

On the other hand some hopes about a regular solutions may stem from the spin-spin repulsion effect of rotating bodies (eventually coupled with the repulsion electomagnetic effect). But because of the increasing technical complexity, the available stationary and rotating solutions involving more than one black hole, as the double Kerr-(Newman) solutions of [4] or [5], just to cite some famous cases, are even more rare than the static 
case. But generally these solutions are neither everywhere regular ${ }^{1}$ nor the two sources are causally connected, as in the case of accelerating and rotating metrics belonging to the Plebanski-Demianki family [10]. Between these kind of metrics the more physical are the ones where the conical singularities lie only between the two sources, avoiding to have conical defects extending to spacial infinity, which remains a globally well defined asymptotic region $[11,12]$.

The purpose of this article is to enrich this scenario furnishing an analytical method to add rotation ${ }^{2}$ to static solutions, including multi black hole ones, in the standard theory of general relativity, without the cosmological constant, coupled to Maxwell electromagnetism.

At this scope, in the context of axisymmetric and stationary space-times, some transformations known to add the Newman-Unti-Tamburino (NUT) parameter to a chosen seed solution, such as the Ehlers or the Reina-Treves transformation, are studied, in particular in section 4 . In section 3 we will see, throughout examples, how the known transformations of these kind, in the realm of pure general relativity, present some issues in the presence of the (standard) electromagnetic field.

In section 4 a new transformation able to add NUT charge to axisymmetric and stationary spacetimes, but without the criticalities of the known transformations, is proposed. In section 5 this enanched transformation is exploited to generate a stationary generalisation of the solution found by Alekseev and Belinski [16], which is an analytical exact solution describing a couple of charged black hole at equilibrium. The extremal specialization of the Alekseev-Belinski-NUT spacetime reduce to the Majumdar-Papapetrou [21, 22] metric endowed with an extra NUT parameter.

The easy near horizon geometry of the latter solution allows one to follow the procedure provided by the Kerr/CFT correspondence [31-34] to address the issue of the microscopic entropy of the Majumdar-Papapetrou black holes.

Since these metrics can describe an ensemble of multiple gravitational sources endowed with electromagnetic monopoles or, thanks to a specific choice of its parameters, a single charged source; it is possible to discuss the second law of black hole thermodynamics: which configuration is favoured from a thermodynamics point of view, specifically which of the two qualitative configurations is more likely to occur as a final state of a gravitational interaction. This is addressed in section 5 .

\section{Review of the Ernst generating technique}

In this article we will focus on the standard theory of General Relativity coupled with Maxwell electromagnetism, governed by the following action

$$
I\left[g_{\mu \nu}, A_{\mu}\right]:=\frac{1}{16 \pi G} \int d^{4} x \sqrt{-g}\left[\mathrm{R}-\frac{G}{\mu_{0}} F_{\mu \nu} F^{\mu \nu}\right] .
$$

\footnotetext{
${ }^{1}$ For a very recent review of the problem see [6].

${ }^{2}$ The rotation we intend here does not refer to the angular momentum, such as the one possessed by the Kerr Black Hole. But we mean the rotation related to others orders in the angular multipole moments expansion, such the ones appearing in NUT solutions. Further details can be found in the section 5.1 and in the appendix B.
} 
From (2.1) it is possible to derive the field equations for the metric $g_{\mu \nu}$ and electromagnetic vector potential $A_{\mu}$

$$
\begin{aligned}
\mathrm{R}_{\mu \nu}-\frac{\mathrm{R}}{2} g_{\mu \nu} & =\frac{2 G}{\mu_{0}}\left(F_{\mu \rho} F_{\nu}{ }^{\rho}-\frac{1}{4} g_{\mu \nu} F_{\rho \sigma} F^{\rho \sigma}\right), \\
\partial_{\mu}\left(\sqrt{-g} F^{\mu \nu}\right) & =0 .
\end{aligned}
$$

The electromagnetic Faraday tensor $F_{\mu \nu}$ is defined, as usual, from the U(1) gauge fourpotential $F_{\mu \nu}:=\partial_{\mu} A_{\nu}-\partial_{\nu} A_{\mu}$. The most generic axisymmetric and stationary spacetime, containing two commuting Killing vectors $\partial_{t}$ and $\partial_{\varphi}$, can be written, for this theory, in the Lewis-Weyl-Papapetrou (LWP) form as

$$
d s^{2}=-f(d t+\omega d \varphi)^{2}+f^{-1}\left[\rho^{2} d \varphi^{2}+e^{2 \gamma}\left(d \rho^{2}+d z^{2}\right)\right] .
$$

All the three structure functions appearing in the metric $f, \omega$ and $\gamma$ depends only on the non-Killing coordinates $(\rho, z)$. We will consider a generic electromagnetic potential compatible with the spacetime symmetries, and the circularity of the LWP metric, given by $A=A_{t}(\rho, z) d t+A_{\varphi}(\rho, z) d \varphi$. For a discussion about the generality of the electromagnetic ansatz see [7].

Ernst in [8] discovered that, when the equations of motion (2.2)-(2.3) are restricted to the above axisymmetric and stationary ansatz, they reduce to a couple of complex vectorial differential equations, as follow ${ }^{3}$

$$
\begin{aligned}
\left(\operatorname{Re} \mathcal{E}+|\boldsymbol{\Phi}|^{2}\right) \nabla^{2} \mathcal{E} & =\left(\vec{\nabla} \mathcal{E}+2 \boldsymbol{\Phi}^{*} \vec{\nabla} \boldsymbol{\Phi}\right) \cdot \vec{\nabla} \mathcal{E} \\
\left(\operatorname{Re} \mathcal{E}+|\boldsymbol{\Phi}|^{2}\right) \nabla^{2} \boldsymbol{\Phi} & =\left(\vec{\nabla} \mathcal{E}+2 \boldsymbol{\Phi}^{*} \vec{\nabla} \boldsymbol{\Phi}\right) \cdot \vec{\nabla} \boldsymbol{\Phi}
\end{aligned}
$$

and two other first order partial differential equations for $\gamma(\rho, z)$, decoupled from the previous ones, (2.5) and (2.6)

$$
\begin{aligned}
\partial_{\rho} \gamma(\rho, z)= & \frac{\rho}{4\left[\operatorname{Re}(\mathcal{E})+\boldsymbol{\Phi} \boldsymbol{\Phi}^{*}\right]^{2}}\left[\left(\partial_{\rho} \mathcal{E}+2 \boldsymbol{\Phi}^{*} \partial_{\rho} \boldsymbol{\Phi}\right)\left(\partial_{\rho} \mathcal{E}^{*}+2 \boldsymbol{\Phi} \partial_{\rho} \boldsymbol{\Phi}^{*}\right)-\left(\partial_{z} \mathcal{E}+2 \boldsymbol{\Phi}^{*} \partial_{z} \boldsymbol{\Phi}\right)\left(\partial_{z} \mathcal{E}^{*}+2 \boldsymbol{\Phi} \partial_{z} \boldsymbol{\Phi}^{*}\right)\right] \\
& -\frac{\rho}{\operatorname{Re}(\mathcal{E})+\Phi \boldsymbol{\Phi}^{*}} \quad\left(\partial_{\rho} \boldsymbol{\Phi} \partial_{\rho} \boldsymbol{\Phi}^{*}-\partial_{z} \boldsymbol{\Phi} \partial_{z} \boldsymbol{\Phi}^{*}\right), \\
\partial_{z} \gamma(\rho, z)= & \frac{\rho}{4\left[\operatorname{Re}(\mathcal{E})+\boldsymbol{\Phi} \boldsymbol{\Phi}^{*}\right]^{2}}\left[\left(\partial_{\rho} \mathcal{E}+2 \boldsymbol{\Phi}^{*} \partial_{\rho} \boldsymbol{\Phi}\right)\left(\partial_{z} \mathcal{E}^{*}+2 \boldsymbol{\Phi} \partial_{z} \boldsymbol{\Phi}^{*}\right)+\left(\partial_{z} \mathcal{E}+2 \boldsymbol{\Phi}^{*} \partial_{z} \boldsymbol{\Phi}\right)\left(\partial_{\rho} \mathcal{E}^{*}+2 \boldsymbol{\Phi} \partial_{\rho} \boldsymbol{\Phi}^{*}\right)\right] \\
& -\frac{\rho}{\operatorname{Re}(\mathcal{E})+\boldsymbol{\Phi} \boldsymbol{\Phi}^{*}} \quad\left(\partial_{\rho} \boldsymbol{\Phi} \partial_{z} \boldsymbol{\Phi}^{*}+\partial_{z} \boldsymbol{\Phi} \partial_{\rho} \boldsymbol{\Phi}^{*}\right) .
\end{aligned}
$$

The complex Ernst potential are defined as

$$
\boldsymbol{\Phi}:=A_{t}+i \tilde{A}_{\varphi}, \quad \mathcal{E}:=f-\mathbf{\Phi} \boldsymbol{\Phi}^{*}+i h,
$$

where $\tilde{A}_{\varphi}$ and $h$ can be obtained from

$$
\begin{aligned}
\vec{\nabla} \tilde{A}_{\varphi} & :=-f \rho^{-1} \vec{e}_{\varphi} \times\left(\vec{\nabla} A_{\varphi}-\omega \vec{\nabla} A_{t}\right) \\
& \vec{\nabla} h:=-f^{2} \rho^{-1} \vec{e}_{\varphi} \times \vec{\nabla} \omega-2 \operatorname{Im}\left(\boldsymbol{\Phi}^{*} \vec{\nabla} \boldsymbol{\Phi}\right) .
\end{aligned}
$$

\footnotetext{
${ }^{3}$ Henceforward in the paper the ratio between the Newton constant and the vacuum permeability is fixed $G / \mu_{0}=1$ without loosing generality.
} 
So the Ernst equations constitute the main equations for the physical system, because once the Ernst potential, satisfying (2.5) and (2.6), are known, $\gamma(\rho, z)$ is obtained by quadratures from (2.7)-(2.8).

The differential operators $\left(\vec{\nabla}\right.$ and $\left.\nabla^{2}\right)$ appearing in $(2.5)-(2.6)$ are just the flat gradient and Laplacian in cylindrical Weyl coordinates $(\rho, z, \varphi){ }^{4}$

The equations of motion for the stationary and axisymmetric complex Ernst potentials (2.5) and (2.6) can be deduced from the following effective action for the complex fields couple $(\mathcal{E}, \boldsymbol{\Phi})$

$$
I(\mathcal{E}, \boldsymbol{\Phi})=\int d z \int d \rho\left[\frac{\left(\vec{\nabla} \mathcal{E}+2 \boldsymbol{\Phi}^{*} \vec{\nabla} \boldsymbol{\Phi}\right)\left(\vec{\nabla} \mathcal{E}^{*}+2 \boldsymbol{\Phi} \vec{\nabla} \boldsymbol{\Phi}^{*}\right)}{\left(\mathcal{E}+\mathcal{E}^{*}+2 \boldsymbol{\Phi} \boldsymbol{\Phi}^{*}\right)^{2}}-\frac{\vec{\nabla} \boldsymbol{\Phi} \vec{\nabla} \boldsymbol{\Phi}^{*}}{\mathcal{E}+\mathcal{E}^{*}+2 \boldsymbol{\Phi} \boldsymbol{\Phi}^{*}}\right]
$$

From the above Lagrangian density in the square brackets, it's possible to derive that the Ernst equations for the complex fields $(\mathcal{E}, \boldsymbol{\Phi})$ have some remarkable non-trivial Lie point symmetries properties $[40,41]$ which form the $\mathrm{SU}(2,1)$ group. These symmetries can be written as a set of five independent transformation which leave invariant the action (2.12) and its equation of motion (2.5)-(2.6):

$$
\begin{aligned}
(I) & \mathcal{E} \longrightarrow \mathcal{E}^{\prime}=\lambda \lambda^{*} \mathcal{E}, & \boldsymbol{\Phi} \longrightarrow \boldsymbol{\Phi}^{\prime}=\lambda \boldsymbol{\Phi}, \\
(I I) & \mathcal{E} \longrightarrow \mathcal{E}^{\prime}=\mathcal{E}+i b, & \boldsymbol{\Phi} \longrightarrow \boldsymbol{\Phi}^{\prime}=\boldsymbol{\Phi}, \\
(I I I) & \mathcal{E} \longrightarrow \mathcal{E}^{\prime}=\frac{\mathcal{E}}{1+i c \mathcal{E}}, & \boldsymbol{\Phi} \longrightarrow \boldsymbol{\Phi}^{\prime}=\frac{\boldsymbol{\Phi}}{1+i c \mathcal{E}} \\
(I V) & \mathcal{E} \longrightarrow \mathcal{E}^{\prime}=\mathcal{E}-2 \beta^{*} \boldsymbol{\Phi}-\beta \beta^{*}, & \boldsymbol{\Phi} \longrightarrow \boldsymbol{\Phi}^{\prime}=\mathbf{\Phi}+\beta, \\
(V) & \mathcal{E} \longrightarrow \mathcal{E}^{\prime}=\frac{\mathcal{E}}{1-2 \alpha^{*} \boldsymbol{\Phi}-\alpha \alpha^{*} \mathcal{E}}, & \boldsymbol{\Phi} \longrightarrow \boldsymbol{\Phi}^{\prime}=\frac{\boldsymbol{\Phi}+\alpha \mathcal{E}}{1-2 \alpha^{*} \boldsymbol{\Phi}-\alpha \alpha^{*} \mathcal{E}}
\end{aligned}
$$

where $b, c \in \mathbb{R}$ and $\alpha, \lambda, \beta \in \mathbb{C}$. Some of these transformation are just gauge symmetries and can be reabsorbed by a coordinate transformation, while others actually have non-trivial physical effects. The combination of $(\mathrm{I})-(\mathrm{V})$ generate other transformation, for example applying (I)-(III) in a certain limit of the parameters ${ }^{5}$ gives the inversion transformation

$$
(\text { inv }) \quad \mathcal{E} \longrightarrow \mathcal{E}^{\prime}=\frac{1}{\mathcal{E}}, \quad \boldsymbol{\Phi} \longrightarrow \boldsymbol{\Phi}^{\prime}=\frac{\boldsymbol{\Phi}}{\mathcal{E}}
$$

which will be useful in the next sections. A particular specialization, for null electromagnetic field, of this inversion transformation is known as the Buchdahl transformation.

In this article we will mainly focus on the transformation of type (III) called the Ehlers transformation. It maps a given solution of the axisymmetric and stationary EinsteinMaxwell equations, identified by the Ernst potentials $(\mathcal{E}, \boldsymbol{\Phi})$, in another non-equivalent one $\left(\mathcal{E}^{\prime}, \boldsymbol{\Phi}^{\prime}\right)$. The Ehlers transformation is parametrised by a real number $c$ which introduce

\footnotetext{
${ }^{4}$ In appendix A some notation on differential operator in various coordinates can be found.

${ }^{5}$ It is easy to verify that the limit for $b \rightarrow \infty$ of the product of the transformations $(I) \circ(I I I) \circ(I I)$ for $c=b^{-1}$ and $\lambda=i b^{-1}$ leads to (2.14) [40].
} 
an extra parameter to the seed solution usually interpreted as the NUT (Newman-UntiTamburino) charge. ${ }^{6}$

Recently the physical significance of the NUT generalisation of lorenzian black holes solutions in general relativity has been partially rehabilitated, under certain assumptions [29]. Nevertheless there is a open discussion about the physical interpretation of the singularities, that may take place in the presence of the NUT parameter. In fact the nodal singularities typically appearing on the axis of symmetry, usually called Misner strings, can be removed by a periodic time identification, which naturally generates closed timelike curves. Therefore some people prefers to keep the string and interpret it as a singular material source of angular momentum.

In [42] the Ehlers transformation, although in another form, was applied to the Kerr black hole seed to obtain the Kerr-NUT spacetime. However we will make use of the Ehlers transformation in the form of (2.13)-(III) because it is easier to apply it to generic Ernst seed potentials.

\section{Example: adding NUT to the Kerr-Newman black hole}

In this section, as an example to show how the solution generating tecnhique works, we will generalise the work of [42] to show how to obtain the Kerr-Newman-NUT solution from the Kerr Newman black hole.

The Kerr-Newman spacetime describes a rotating and charged asymptotically flat black hole in the theory of general relativity. In the presence of both electric and magnetic monopole charge, respectively labelled $q$ and $p$, the solution can be written in BoyerLindquist coordinates as follows

$$
\begin{aligned}
d s^{2} & =-\frac{\Delta(r)}{R(r, \theta)^{2}}\left(d t-a \sin ^{2} \theta d \varphi\right)^{2}+R(r, \theta)^{2}\left(\frac{d r^{2}}{\Delta(r)}+d \theta\right)+\frac{\sin ^{2} \theta}{R(r, \theta)^{2}}\left[a d t-\left(r^{2}+a^{2}\right) d \varphi\right]^{2}, \\
A_{\mu} & =\left[\frac{q r+p a \cos \theta}{R(r, \theta)^{2}}, 0,0, \frac{p \cos \theta\left(r^{2}+a^{2}\right)-a q r \sin ^{2} \theta}{R(r, \theta)^{2}}\right]
\end{aligned}
$$

where

$$
R(r, \theta):=r^{2}+a^{2} \cos ^{2} \theta, \quad \Delta(r):=r^{2}-2 m r+a^{2}+q^{2}+p^{2} .
$$

\footnotetext{
${ }^{6}$ The term charge is possibly abused in this context because the NUT parameter is not actually related to a conserved quantity associated to a physical symmetry, as it occurs for the mass or the angular momentum. But still this terminology is often used in the literature, usually referring to a topological invariant quantity that can be associated to the NUT parameter, similarly to the magnetic monopole charge [20]. In fact, from the analogy with the duality between the electric and magnetic monopoles charges, the NUT parameter is usually denoted as the gravitomagnetic monopole mass, as the dual to the standard gravitational monopole mass. This because the mass parameter mainly contributes to the electric part of the Weyl tensor decomposition, while the NUT parameter to the magnetic sector. Physically this property is related to the fact that the NUT parameter introduces an interaction on trajectories of massive test particles similar to the magnetic force on a charged particle, even if it is a purely gravitational solution. For this reason sometimes in the literature the NUT parameter is associated to radial angular momentum to distinguish it from standard axial angular momentum of the Kerr black hole.
} 
The first step of the Ernst procedure consists in identifying the $f, \omega, \gamma$ functions appearing in the Lewis-Weyl-Papapetrou ansatz (2.4) for the seed metric, which in this case is the Kerr-Newman one (3.1). At this purpose the coordinates transformation

$$
\rho(r, \theta):=\sin \theta \sqrt{\Delta(r)}, \quad z(r, \theta):=\cos \theta(r-m)
$$

is applied to (2.4) to get a better suited LWP line element for the seed coordinates

$$
d s^{2}=-f(r, \theta)[d t+\omega(r, \theta) d \varphi]^{2}+\frac{1}{f(r, \theta)}\left\{e^{2 \gamma(r, \theta)}\left[(r-m)^{2}-\kappa^{2} \cos ^{2} \theta\right]\left[\frac{d r^{2}}{\Delta(r)}+d \theta^{2}\right]+\sin ^{2} \theta \Delta(r) d \varphi^{2}\right\} .
$$

The constant $\kappa$, specifically for the Kerr-Newman spacetime, takes the value $\kappa=$ $\sqrt{m^{2}-a^{2}-q^{2}-p^{2}}$.

By comparing the metrics (3.1) and (3.5) it is possible to determinate the structure functions of the Kerr-Newman metric. We will present them in more ergonomic coordinates $(r, x:=\cos \theta)$ :

$$
\begin{aligned}
f_{0}(r, x) & =1+\frac{q^{2}+p^{2}-2 m r}{r^{2}+a^{2} x^{2}} \\
\omega_{0}(r, x) & =\frac{a\left(1-x^{2}\right)\left(2 m r-q^{2}-p^{2}\right)}{2\left(r^{2}-2 m r+q^{2}+p^{2}+a^{2} x^{2}\right)} \\
e^{2 \gamma_{0}}(r, x) & =\frac{r^{2}-2 m r+q^{2}+p^{2}+a^{2} x^{2}}{(r-m)^{2}-\kappa^{2} x^{2}} .
\end{aligned}
$$

The differential operators $\vec{\nabla}$ and $\nabla^{2}$ in terms of the coordinates $(r, x)$ becomes

$$
\begin{aligned}
\vec{\nabla} \phi(r, x) & =\frac{1}{\sqrt{(r-m)^{2}-\kappa^{2} x^{2}}}\left[\vec{e}_{r} \sqrt{\Delta(r)} \frac{\partial \phi(r, x)}{\partial r}+\vec{e}_{x} \sqrt{1-x^{2}} \frac{\partial \phi(r, x)}{\partial x}\right] \\
\nabla^{2} \phi(r, x) & =\frac{1}{(r-m)^{2}-\kappa^{2} x^{2}}\left\{\frac{\partial}{\partial r}\left[\Delta(r) \frac{\partial \phi(r, x)}{\partial r}\right]+\frac{\partial}{\partial x}\left[\left(1-x^{2}\right) \frac{\partial \phi(r, x)}{\partial x}\right]\right\} .
\end{aligned}
$$

The $(r, x)$ coordinates are closely related with the prolate spherical ones $(y, x)$. To obtain these latter is sufficient to define $y:=(r-m) / \kappa$.

Then in order to identify the electromagnetic seed Ernst potential $\mathbf{\Phi}_{0},{ }^{7}$ as defined in (2.9), for the Kerr-Newman gauge field we need to derive $\tilde{A}_{\varphi}$ from eq. (2.10) and taking into account eqs. (3.2), (3.6)-(3.9). For the seed under consideration we have

$$
\tilde{A}_{\varphi 0}(r, x)=\frac{a q x-p r}{r^{2}+a^{2} x^{2}} \quad \Longrightarrow \quad \Phi_{0}(r, x)=-\frac{q+i p}{r+i a x} .
$$

While to obtain the gravitational Ernst potential for the seed Kerr-Newman metric $\mathcal{E}_{0}$ we have first to integrate (2.11) to get

$$
h_{0}(r, x)=\frac{2 m x}{r^{2}+a^{2} x^{2}} \quad \Longrightarrow \quad \mathcal{E}_{0}(r, x)=1-\frac{2 m}{r+i a x} .
$$

\footnotetext{
${ }^{7}$ The zero subscript in $\boldsymbol{\Phi}_{0}$ point out that we refer to the seed fields.
} 
Now we can apply the Ehlers transformation (2.13)-(III) to the seed Ernst potentials $\left(\mathcal{E}_{0}, \mathbf{\Phi}_{0}\right)$ to generate a new axisymmetric and stationary solution of the Einstein-Maxwell theory in terms of the Ernst potentials

$$
\begin{gathered}
\mathcal{E}(r, x)=\frac{\mathcal{E}_{0}(r, x)}{1+i c \mathcal{E}_{0}(r, x)}=\frac{a x+2 i m-i r}{a x-i r+c(r-2 m+i a x)}, \\
\boldsymbol{\Phi}(r, x)=\frac{\boldsymbol{\Phi}_{0}(r, x)}{1+i c \mathcal{E}_{0}(r, x)}=\frac{-p+i q}{a x-i r+c(r-2 m+i a x)} .
\end{gathered}
$$

To come back to the metric and vector potential representation it is sufficient to use the definitions (2.9)-(2.11). In particular $f, \omega, A_{t}$ can be read directly from (2.9)

$$
\begin{gathered}
h(r, x)=\operatorname{Im}(\mathcal{E})=\frac{2 a m x-c\left[\left(r^{2}-2 m\right)^{2}+a^{2} x^{2}\right]}{r^{2}-4 a c m x+a^{2} x^{2}+c^{2}\left[\left(r^{2}-2 m\right)^{2}+a^{2} x^{2}\right]}, \\
f(r, x)=\operatorname{Re}(\mathcal{E})+\boldsymbol{\Phi} \boldsymbol{\Phi}^{*}=\frac{p^{2}+q^{2}-2 m r+r^{2}+a^{2} x^{2}}{r^{2}-4 a c m x+a^{2} x^{2}+c^{2}\left[\left(r^{2}-2 m\right)^{2}+a^{2} x^{2}\right]}, \\
A_{t}(r, x)=\operatorname{Re}(\boldsymbol{\Phi})=\frac{-q r-a p x+c(2 m p-p r+a q x)}{r^{2}-4 a c m x+a^{2} x^{2}+c^{2}\left[\left(r^{2}-2 m\right)^{2}+a^{2} x^{2}\right]}, \\
\tilde{A}_{\varphi}(r, x)=\operatorname{Im}(\boldsymbol{\Phi})=\frac{-2 c m q-p r+c q r+a(c p+q)}{r^{2}-4 a c m x+a^{2} x^{2}+c^{2}\left[\left(r^{2}-2 m\right)^{2}+a^{2} x^{2}\right]} .
\end{gathered}
$$

The above quantities have to be inserted in the equation (2.11) to obtain

$$
\omega(r, x)=-4 c m x+\frac{a\left(x^{2}-1\right)\left\{p^{2}+q^{2}-2 m r+c^{2}\left[p^{2}+q^{2}+2 m(r-2 m)+4 a c m x\right]\right\}}{r^{2}-2 m r+q^{2}+p^{2}+a^{2} x^{2}}+\omega_{0} .
$$

Then, finally, also (2.10) can be solved to get

$$
A_{\varphi}(r, x)=p x-c q x-\frac{[-2 c m p+c p r+q r+a(p-c q) x]\left[-4 c m x+a\left(1+c^{2}\right)\left(x^{2}-1\right)+\omega_{0}\right]}{r^{2}-4 a c m x+a^{2} x^{2}+c^{2}\left[(r-2 m)^{2}+a^{2} x^{2}\right]}+A_{\varphi 0} .
$$

The arbitrary constants $\omega_{0}$ and $A_{\varphi 0}$ usually are constrained by asking regularity of the metric and the magnetic field on the symmetry axis $\rho=0$. In particular the magnetic field, to be globally well behaved, should fulfil the physical requirement assuring that the magnetic monopole moments must be null on the axis of symmetry [45], therefore

$$
\lim _{\rho \rightarrow 0} A_{\varphi}(\rho, z)=0 .
$$

The Ehlers transformation is not affecting the $\gamma$ function, which remains the same as the seed $\gamma_{0}$. This can be directly verified, substituting (3.13)-(3.14) into eqs. (2.7)-(2.8).

The solution generated by the Ehlers transformation represents a Kerr-Newman black hole in a Taub-NUT background, whose electromagnetic vector potential is generally written in this form

$$
\hat{A}_{\mu}=\left\{-\frac{q r+p(a x+\ell)}{r^{2}+(\ell+a x)^{2}}, 0,0,-q \frac{r(x-1)(a+a x+2 \ell)}{r^{2}+(\ell+a x)^{2}}+p \frac{(a x+\ell)\left[r^{2}+(a+\ell)^{2}\right]}{a\left[r^{2}+(\ell+a x)^{2}\right]}-\hat{\omega}_{0} \hat{A}_{t}(r, x)+\hat{A}_{\varphi 0}\right\} .
$$


To have a well defined Maxwell potential, in the no rotation limit, the constant $\hat{A}_{\varphi 0}$ have to be fiexd to $-\frac{p \ell}{a}$.

In the case one wants to verify the equivalence of the generated metric and the standard Kerr-Newman-NUT one, denoted as $d \hat{s}^{2}$, it is necessary a coordinate transformation and a rescaling some parameters.

Interesting enough the same procedure is not sufficient to get the electromagnetic potential $\hat{A}_{\mu}$ of eq. (3.22), in fact neither the asymptotic behaviour of the RN-NUT electromagnetic field is retrieved. A further duality transformation on the electromagnetic field is required. Of course in four-dimensions this transformation is not affecting the metric. In terms of the Ernst potential the duality transformation can be written as

$$
\boldsymbol{\Phi} \longrightarrow \overline{\boldsymbol{\Phi}}=\boldsymbol{\Phi} \exp (i \beta)
$$

Actually this is a special unitary sub-case, when $\lambda=\exp (i \beta)$, of the more general symmetry (2.13)-(I) of the Ernst electrovacuum equations (2.5)-(2.6).

Thus the non-null components of the rotated electromagnetic vector potential (3.17) and (3.20) can be obtained by (2.9) and (2.10). They become respectively

$$
\begin{aligned}
& \bar{A}_{t}(r, x)=\frac{-[-2 c m p+c p r+q r+a x(p-c q)] \cos \beta+[2 c m q+p r-c q r-a x(c p+p)] \sin \beta}{r^{2}-4 a c m x+a^{2} x^{2}+c^{2}\left[(r-2 m)^{2}+a^{2} x^{2}\right]}, \\
& \bar{A}_{\varphi}(r, x)=(p-c q) x \cos \beta+(c p+q) x \sin \beta-\left[-4 c m x+a\left(1+c^{2}\right)\left(x^{2}-1\right)+\omega_{0}\right] \bar{A}_{t}(r, x) .
\end{aligned}
$$

To check the equivalence of the electromagnetic potentials (3.24)-(3.25) and (3.22) the following coordinates transformation of the temporal and radial coordinate is needed ${ }^{8}$

$$
r \longrightarrow \bar{r}=r \sqrt{1+c^{2}}-\frac{2 c^{2} m}{\sqrt{1+c^{2}}}, \quad t \longrightarrow \bar{t}=\frac{t}{\sqrt{1+c^{2}}},
$$

and a rescaling of the physical parameters ${ }^{9}$

$$
\begin{array}{lrl}
q \longrightarrow \bar{q}=q \sqrt{1+c^{2}}, & p \longrightarrow \bar{p}=p \sqrt{1+c^{2}}, \\
a \longrightarrow \bar{a}=a \sqrt{1+c^{2}}, & m \longrightarrow \bar{m}=-\frac{\ell \sqrt{1+c^{2}}}{2 c} .
\end{array}
$$

Where the relation between the extra parameter introduced by the Ehlers transformation $c$ and the standard NUT one $\ell$ is

$$
c=\frac{m-\sqrt{m^{2}+\ell^{2}}}{\ell^{2}} .
$$

Finally the particular duality rotation (3.23), which completes the alignment of the electromagnetic vector potentials $\bar{A}_{\mu}$ and $\hat{A}_{\mu}$, is given by

$$
\cos \beta=\frac{1}{\sqrt{1+c^{2}}}=\sqrt{\frac{1}{2}+\frac{m}{2 \sqrt{m^{2}+\ell^{2}}}} .
$$

${ }^{8} \hat{\omega}_{0}$ and $\hat{A}_{\varphi 0}$ are usually chosen to be null, while $\omega_{0}=-2 \ell$.

${ }^{9}$ This parametrization is consistent for the negative branch of $\ell$, when $\ell>0$ some signs change. 
Thanks to the above coordinates transformation also the Ehlers transformed Kerr-Newman metric, basically determined by eqs. (2.4), (3.8), (3.16) and (3.19), fits into the usual dyonic Kerr-Newmann-NUT form

$$
\hat{d s}^{2}=-\hat{f}(d t+\hat{\omega} d \varphi)^{2}+\hat{f}^{-1}\left[\alpha^{2} d \varphi^{2}+e^{2 \hat{\gamma}}\left(\frac{d r^{2}}{Q(r)}+\frac{d x^{2}}{P(x)}\right)\right] .
$$

where

$$
\begin{aligned}
\hat{f}(r, x) & =\frac{Q(r)-a^{2} P(x)}{r^{2}+(\ell+a x)^{2}}, \\
\hat{\omega}(r, x) & =-\frac{a\left[r^{2}+(a+l)^{2} P(x)\right]+(x-1)(a+a x+2 \ell) Q(r)}{a^{2} P(x)-Q(r)}-\hat{\omega}_{0}, \\
\alpha(r, x) & =\sqrt{Q(r) P(x)}, \\
\hat{\gamma}(r, x) & =Q(r)-a^{2} P(x), \\
Q(r) & =r^{2}-2 m r+a^{2}+q^{2}+p^{2}-\ell^{2}, \\
P(x) & =1-x^{2} .
\end{aligned}
$$

This procedure is completely generic and does not apply only to the Kerr-Newman spacetime, but to all axisimmetric and stationary spacetimes of Einstein-Maxwell theory.

In the section 5 the same technique will be exploited to obtain a new solution describing a NUT generalisation of a couple of charged black holes, but through a slightly different Ehlers transformation, which will be described in the next section.

\section{Enhanced Ehlers transformations and gravitomagnetic duality}

Originally Ehlers, in his 1959 thesis, discovered a symmetry of the Ernst field equations (2.5)-(2.6) that can be written as follows

$$
\left\{\begin{aligned}
U+W & \longrightarrow U+W+i c(U-W) \\
V & \longrightarrow V \\
U-W & \longrightarrow U-V
\end{aligned}\right.
$$

for any complex Ernst potential of the form

$$
\mathcal{E}=\frac{U-W}{U+W}, \quad \boldsymbol{\Phi}=\frac{V}{U+W} .
$$

It easy to show that the (4.1) transformation is equivalent to the Ehlers transformation written in the form of eq. (2.13)-(III). Nevertheless in the previous section we have realised that, in general, the so called Ehlers transformation given in eqs. (2.13)-(III) is not sufficient to add the NUT charge to the electrovacuum axisymmetric spacetime seed. When the seed presents a non-null Maxwell electromagnetic field, the Ehlers transformation (2.13)-(III) adds an additional (and undesired) rotation to the $\mathrm{U}(1)$ electromagnetic vector potential and an extra duality transformation of the Ernst potential is needed. 
On the other hand Reina and Treves in [42] pointed out how to add NUT charge to spacetimes whose Ernst complex potentials can be written as

$$
\mathcal{E}=\frac{\xi-1}{\xi+1}, \quad \boldsymbol{\Phi}=\frac{q}{\xi+1} .
$$

As explained by Ernst in [8], the above form for the complex potentials stems, from the original one, as defined in (2.9), requiring that $\mathcal{E}$ is an analytic function of $\boldsymbol{\Phi}$ and using boundary conditions which mimic the asymptotic flatness: $\mathcal{E}=1$ and $\boldsymbol{\Phi}=0$ at spatial infinity. In fact, as the Kerr-Newman solution fulfil these requirements, it can be cast into the form (4.3). Actually in [42] only uncharged solutions were treated such as the Kerr black hole or the Tomimatsu-Sato metric. Anyway it can be shown that the NUT parameter can be added to the seed spacetime written as (4.3), just rotating the complex function $\xi$ by a constant phase in the complex plane

$$
\xi \longrightarrow \bar{\xi}=\xi e^{i \tau} .
$$

However in presence of the electromagnetic potential, just as for the Ehlers transformation, the procedure is not so straight.

First of all we would like to clarify the relation between the Ehlers transformation and the Reina-Treves one (4.4), apart the mentioned reduced domain of applicability of the latter. Applying the following transformations to the complex Ernst potentials (4.3)

$$
(i n v) \circ(I I) \circ(I) \circ(i n v) \circ(I I) \circ\left(\begin{array}{c}
\mathcal{E} \\
\Phi
\end{array}\right)=\left(\begin{array}{c}
\overline{\mathcal{E}} \\
\overline{\mathbf{\Phi}}
\end{array}\right),
$$

and considering $\lambda=1-i b$ we get the transformed complex potentials

$$
\overline{\mathcal{E}}=\frac{\xi(1+i b)-(1-i b)}{\xi(1+i b)+(1-i b)}, \quad \bar{\Phi}=\frac{q(1-i b)}{\xi(1+i b)+(1-i b)} .
$$

Finally defining $\tau:=\arccos \left(\frac{1-b^{2}}{1+b^{2}}\right)$ the above Ernst potentials transform in

$$
\overline{\mathcal{E}}=\frac{\xi e^{i \tau}-1}{\xi e^{i \tau}+1}, \quad \overline{\mathbf{\Phi}}=\frac{q}{\xi e^{i \tau}+1},
$$

which exactly correspond to the transformation given in (4.4), when applied to the potentials of the form (4.3). Therefore we have shown how the Reina-Treves transformation can be deduced from a proper composition of the basic invariance symmetries (2.13) of the Ernst Fields equations. Note that the relation between the Reina-Treves and Ehlers transformation relies on the fact that, the latter is a part of the inverse transformation we used in eq. (4.5), as explained in footnote 5.

Now that we know how to obtain the Reina-Treves transformation from the basic $\mathrm{SU}(2,1)$ symmetries transformations (2.13), we can extend the transformation (4.4) for more general Ernst potentials with respect to the asymptotic flat subclass (4.3) of the Reina-Treves. Just applying the sequence of transformations (4.5) to unconstrained Ernst potentials we get

$$
\mathcal{E} \longrightarrow \overline{\mathcal{E}}=\frac{\mathcal{E}+i b}{1+i b \mathcal{E}}, \quad \boldsymbol{\Phi} \longrightarrow \overline{\boldsymbol{\Phi}}=\frac{\boldsymbol{\Phi}(1-i b)}{1+i b \mathcal{E}} .
$$


Note that, when the Reina-Treves transformation is written in this generalised form, the similarities with the Ehlers transformation are quite apparent.

Nevertheless, repeating the procedure discussed in the previous section for the Ehlers transformation, but this time using the transformation proposed by Reina-Treves, we realise that also in this case an extra duality transformation is needed in the presence of the electromagnetic field (followed by a coordinates transformation ${ }^{10}$ too).

Instead it would be optimal to know exactly which is the transformation able to only add the NUT charge to a chosen seed spacetime. This point turns out to be particularly relevant, as in the next section case, where the result of the transformation is an unknown solution, thus in principle we ignore if extra manipulations or transformations are needed to get only the NUT extension (and eventually which ones).

After having repeated the previous section algorithm to add the NUT charge to the Kerr-Newman black hole for the Reina-Treves transformation (4.7) or for its generalised version (4.8), ${ }^{11}$ it is possible to understand which kind of duality transformation of the electromagnetic field is necessary. In fact the actual transformations which produces the NUT generalisation of a given seed spacetime is

$$
\mathcal{E} \longrightarrow \overline{\mathcal{E}}_{N}=\frac{\mathcal{E}+i b}{1+i b \mathcal{E}}, \quad \boldsymbol{\Phi} \longrightarrow \overline{\mathbf{\Phi}}_{N}=\frac{\boldsymbol{\Phi}(1+i b)}{1+i b \mathcal{E}} .
$$

Otherwise using the Reina-Treves original notation of the article [42] the enhanced transformation reads

$$
\mathcal{E} \longrightarrow \overline{\mathcal{E}}=\frac{\xi e^{i \tau}-1}{\xi e^{i \tau}+1}, \quad \boldsymbol{\Phi} \longrightarrow \overline{\boldsymbol{\Phi}}=\frac{q e^{i \tau}}{\xi e^{i \tau}+1} .
$$

The gravitational part of the enhanced Ehlers transformation (4.9) is compatible with the one presented in [20], but there electromagnetic fields were not considered.

Moreover the transformation (4.9) provides better asymptotic properties to the Ernst potentials with respect to the standard Ehlers transformation (2.13)-(III). In fact, the generic asymptotic expansion of the Ersnt complex potentials for asymptotically flat metrics (possibly enlarged by the presence of the Taub-NUT parameter) in terms of large radial coordinate $r$, as presented in [28], is

$$
\begin{aligned}
& \mathcal{E} \sim 1-\frac{2(M-i B)}{r}+\frac{\left(z_{*}+2 i J\right) y+\text { const. }}{r^{2}}+O\left(\frac{1}{r^{3}}\right), \\
& \boldsymbol{\Phi} \sim \frac{Q_{e}+i Q_{m}}{r}+\frac{\left(D_{e}+i D_{m}\right) y+\text { const. }}{r^{2}}+O\left(\frac{1}{r^{3}}\right),
\end{aligned}
$$

where $M, B, J, Q_{e}, Q_{m}, D_{e}, D_{m}$, respectively identify the total conserved quantities: mass, NUT, angular momentum, total electric and magnetic charge, electric and magnetic dipole moments, while $z_{*}$ is a constant related to the position of the origin of the quasi-spherical coordinates $(r, \theta)$.

\footnotetext{
${ }^{10}$ Note that coordinate transformations and the symmetries of the Ernst potentials (2.13) do not commute in general. Therefore, in order to have some control on the resulting spacetime, when several consecutive transformations are composed it is better to avoid coordinate transformations.

${ }^{11}$ In appendix B the main results of this "procedure" are summarised.
} 
It can be easily checked, for instance though the Kerr-Newman-NUT examples of section 3, that the standard Ehlers transformation (2.13)-(III) or (3.13)-(3.14) is not compatible with the asymptotic fields fall-off at spatial infinity, instead the enhanced Ehlers transformation (4.9), in the context of the Kerr-Newman-NUT solution it is explicitly given in eqs. (B.1)-(B.2), perfectly fulfil the expected decaying of the gravitational and electromagnetic complex potentials. In practice it means that the enhanced Ehlers transformation does not change the values of the other conserved charges, such as electric or magnetic charges, of the given seed spacetime as all the previous variants of the Ehlers transformation do. Thus the enhanced transformation adds the NUT charge while preserving the original physical properties of the initial solution. ${ }^{12}$ Further evidences of the asymptotic superiority of the enhanced Ehlers transformation are presented in the context of multiple gravitational sources, in section 5.2.

The disadvantages of the traditional form of the Ehlers transformation are usually fixed with additional ad-hoc transformations or supplementary boundary conditions. On the contrary, in the examples considered in this paper, both with single and double gravitational sources, the enhanced Ehlers transformation, by construction, does not mix the electric with the magnetic field.

Basically, since the enhanced Ehlers transformation, when applied to asymtotically flat spacetimes (as can be also understood from the Kerr-Newman example), preserves the Ernst field fall-off (4.11)-(4.12), it can be considered as the analogous of the electromagnetic duality transformation, but for the gravitational field. In fact in four dimensions the electromagnetic duality rotates the electric monopole charge into the magnetic one, while preserving the spacetime metric and the physical charges such as the mass, the angular momentum or the nut charge. ${ }^{13}$ Similarly the enhanced Ehlers transformation rotates the mass into the nut charge while preserving the electromagnetic charges; see the appendix B for details in the context of the Kerr-Newman black hole.

Thus the analogy with the electromagnetic duality give us the opportunity to clarify the action of the (enhanced) Ehlers transformation, which effect does not consist merely in adding NUT charge to any axisymmetric and stationary solution. Since the Ehlers transformation rotates the mass charge into the gravomagnetic charge, it means that it is rotating the electric part of the Weyl tensor of the seed metric into it magnetic part. Indeed seed spacetimes with trivial mass charge do not acquire any NUT charge as well, after an Ehlers transformation. This new insight about the role of the Ehlers transformation naturally opens to explore the possibility of having further dualities stemming from the symmetries of the Ernst equations in general relativity. In fact, at least in restricted setting

\footnotetext{
${ }^{12}$ Note that mess in the conserved charges introduced cannot be restored by any coordinate transformation or parameter redefinition.

${ }^{13}$ Very recently, in [43], the gravitomagnetic duality is ascribed to be related to a variant of the JanisNewman trick. Being a complex coordinate transformation, that trick (considered by Ernst a "method which transcend logic" [8]) is not a systematic procedure based on the equations of motion, as the solution generating techniques is. Therefore, contrary to the solution generating technique which by construction maps solutions into solutions, when dealing with the Janis-Newman trick, in general, one has not to expect to obtain new metrics or matter fields which fulfil the equations of motion. In the cases the trick may work, for adding the nut charge, it should reduce to the Ehlers transformation.
} 
of the Plebanski-Demianski class of metrics, it is well known that the NUT parameter is the dual of the mass charge, the electric charge is dual to the magnetic monopole and also the rotation and the acceleration parameter are similarly related [44]. Therefore would be plausible to search for the transformation able to rotate the angular momentum charge into the acceleration parameter, at least in the context of axisymmetric and stationary spacetimes.

To sum up neither the Ehlers nor the Reina-Treves transformations exactly generate the NUT extension of the seed electrovacuum spacetime, but the (4.9) or (4.10) do. Maybe this can be the reason why Reina and Treves in [42] treated only metrics, such as the Kerr black hole or the Tomimatsu-Sato, with no electromagnetic field. Note also that when trivial and non-trivial transformations are sequentially composed, they may be not reabsorbed by a gauge or a coordinate transformation. For instance the inversion transformation maps the trivial transformation (2.13)-(II) into the non trivial (2.13)-(III). Thus, henceforward in the article, we will prefer to work with the transformation (4.9) to build new solutions, because it is more precise, especially when simultaneously composed with others transformations and because it can be applied to general seeds in the presence of the Maxwell electromagnetic field in a more controlled way, so it is more convenient.

\section{$5 \quad$ NUT generalisation of Majumdar-Papapetrou black holes}

Now that we have clarified which is the proper transformation able to add the NUT charge to any axisymmetric and stationary electrovacuum space-time, would be interesting to test its effectiveness by applying the results of the previous section to a given seed to obtain a novel solution.

The generalised enhanced Reina-Treves transformation (4.9) is able to map a static metric in a stationary one. This feature can be exploited to build, for the first time, a coalescence of a binary system composed by two rotating regular black holes at equilibrium. While some binary system describing two Kerr sources at equilibrium have been found [4, $5,12,13]$, it can be shown that these sources can never describe a legit couple of black holes because one of the two sources have to be hyper-extremal [19]. If one insists in keeping both Kerr sources under the extremal limit, non-removable conical singularities between the two black holes, not covered by any horizon, appears. Recently also a couple of regular but accelerating and rotating black holes have been obtained by the Ernst technique [14] but the two sources are of a different kind with respect the above examples, as they are not casually connected.

Possibly the easiest path to reach our goal is to consider one of the simplest binary regular black hole couple, i.e. the Majumdar-Papapetrou solution [21, 22] and its nonextremal generalisations. It describes an ensemble of charged and extremal black holes, of the Reissner-Nordstrom type, at equilibrium, where the gravitational attraction is compensated by the electric repulsion between the sources. While the ensemble can be formed by an arbitrary number of sources, for simplicity we will just focus on the easier case composed by two black holes. 


\subsection{The non-extremal case: NUT generalisation of a RN black holes pair}

Alekseev and Belinski [15] and Manko [17] have been able extend the Majumdar-Papapetrou di-hole solution also beyond the extremal case. But, even in this setting, outside the extremality the two sources, at equilibrium, cannot simultaneously be under-extreme black holes without the introduction of extra conical singularities. Using the notation of [15] the double (non-extremal) Reissner-Nordstrom solution can be written in terms of the LWP metric (2.4), where the structure functions are

$$
\begin{aligned}
f(\rho, z) & :=\frac{\mathcal{D}^{2}-\mathcal{G}^{2}+\mathcal{F}^{2}}{(\mathcal{D}+\mathcal{G})^{2}} \\
\omega(\rho, z) & :=0 \\
e^{2 \gamma(\rho, z)} & :=f_{0} \frac{\mathcal{D}^{2}-\mathcal{G}^{2}+\mathcal{F}^{2}}{\prod_{i=1}^{2}\left(x_{i}^{2}-\sigma_{i}^{2} y_{i}^{2}\right)}
\end{aligned}
$$

with $i \in\{1,2\}$ and

$$
\begin{aligned}
\mathcal{D} & :=x_{1} x_{2}-\bar{\gamma}^{2} y_{1} y_{2}+\delta\left[x_{1}^{2}+x_{2}^{2}-\sigma_{1}^{2} y_{1}^{2}-\sigma_{2} y_{2}^{2}+2\left(m_{1} m_{2}-q_{1} q_{2}\right) y_{1} y_{2}\right] \\
\mathcal{G} & :=m_{1} x_{2}+m_{2} x_{1}+\bar{\gamma}\left(q_{1} y_{1}+q_{2} y_{2}\right)+2 \delta\left[m_{1} x_{1}+m_{2} x_{2}+y_{1}\left(q_{2} \bar{\gamma}-m_{1} l\right)+y_{2}\left(q_{1} \gamma+m_{2} l\right)\right] \\
\mathcal{F} & :=q_{1} x_{2}+q_{2} x_{1}+\bar{\gamma}\left(m_{1} y_{1}+m_{2} y_{2}\right)+2 \delta\left[q_{1} x_{1}+q_{2} x_{2}+y_{1}\left(m_{2} \bar{\gamma}-q_{1} l\right)+y_{2}\left(m_{1} \bar{\gamma}+q_{2} l\right)\right] .
\end{aligned}
$$

The bipolar coordinates $\left(x_{i}, y_{i}\right)$ are defined with respect to the coordinates $(\rho, z)$ as follows

$$
\begin{aligned}
& x_{i}(\rho, z):=\frac{1}{2}\left[\sqrt{\rho^{2}+\left(z-z_{i}+\sigma_{i}\right)^{2}}+\sqrt{\rho^{2}+\left(z-z_{i}+\sigma_{i}\right)^{2}}\right], \\
& y_{i}(\rho, z):=\frac{1}{2 \sigma_{i}}\left[\sqrt{\rho^{2}+\left(z-z_{i}+\sigma_{i}\right)^{2}}-\sqrt{\rho^{2}+\left(z-z_{i}+\sigma_{i}\right)^{2}}\right] .
\end{aligned}
$$

The non extremal solution at equilibrium has four independent parameters, the ones related to the masses and charges ${ }^{14}$ of the two black holes $m_{1}, m_{2}, q_{1}, q_{2}$, constrained by the equilibrium condition $m_{1} m_{2}=q_{1} q_{2}$ and the constant $\bar{\gamma}$, related to the distance $l$ between the two mass sources placed on the $z$-axis at the points $z_{i}$

$$
l=z_{2}-z_{1}=\left(m_{2} q_{1}-m_{1} q_{2}\right) / \bar{\gamma} .
$$

The constants $\sigma_{i}$ are connected to the above parameter $\bar{\gamma}$ by the two constraints $\sigma_{i}=m_{i}^{2}+$ $\bar{\gamma}^{2}-q_{i}^{2}$; they determine the position of the horizons, located at $\left\{\rho=0, z_{i}-\sigma_{i} \leq z \leq z_{i}+\sigma_{i}\right\}$, which distance is given by $l-\sigma_{1}-\sigma_{2}$. While the distance between a naked singularity (associated to the source of mass $m_{1}$ ) and a black hole (associated to the source of mass $\left.m_{2}\right)$ is given by $l-\sigma_{2}$. Other auxiliary constants are

$$
f_{0}:=\frac{1}{(1+2 \delta)^{2}}, \quad \delta:=\frac{m_{1} m_{2}-q_{1} q_{2}}{l^{2}-m_{1}^{2}-m_{2}^{2}+q_{1}^{2}+q_{2}^{2}},
$$

\footnotetext{
${ }^{14}$ Note that $q_{i}$ does not coincide with the effective electric charge of the $i$ black hole, which is, according to $[15] e_{1}=q_{1}-\bar{\gamma}, e_{2}=q_{2}+\bar{\gamma}$, at least in lack of the NUT parameter.
} 
but at equilibrium they become trivial: $f_{0}=1$ and $\delta=0$. The electromagnetic vector potential supporting this metric is

$$
A_{\mu}=\left(\frac{\mathcal{F}}{\mathcal{D}+\mathcal{G}}, 0,0,0\right)
$$

To obtain the NUT extension of this solution first it is necessary to get its Ernst complex potentials. From definitions (2.9) we have

$$
\mathcal{E}=\frac{\mathcal{D}-\mathcal{G}}{\mathcal{D}+\mathcal{G}}, \quad \boldsymbol{\Phi}=\frac{\mathcal{F}}{\mathcal{D}+\mathcal{G}} .
$$

Hence applying the enhanced generalised Reina-Treves transformation (4.10) we can add the NUT charge, parametrised by $b$, to the equilibrium configuration of two ReissnerNordstrom sources. In term of the Ernst potentials it reads

$$
\mathcal{E}_{N}=\frac{\mathcal{D}(1+i b)-\mathcal{G}(1-i b)}{\mathcal{D}(1+i b)+\mathcal{G}(1-i b)}, \quad \mathbf{\Phi}_{N}=\frac{\mathcal{F}(1+i b)}{\mathcal{D}(1+i b)+\mathcal{G}(1-i b)} .
$$

As can be easily understood from the non-null imaginary part of the Ernst potential the static Alekseev-Belinski solution after the above transformation becomes stationary. The structure functions for the metric and Maxwell potential can be deduced from eqs. (2.9)(2.11), as it was done in the Kerr-Newman-NUT case of section 3.

From the asymptotic fall-off of the Ernst fields we can infer the relation between the physical parameters of the seed and of the solution after the Ehlers transformation

$$
\begin{aligned}
b & \longrightarrow \frac{-M+\sqrt{M^{2}+\ell^{2}}}{\ell}, \\
m_{1} & \longrightarrow \frac{\left[q_{1}\left(q_{1}+q_{2}\right)+z_{2}\left(z_{2}-z_{1}\right)\right] \sqrt{M^{2}+\ell^{2}}}{\left(q_{1}+q_{2}\right)^{2}+\left(z_{1}-z_{2}\right)^{2}}, \\
m_{2} & \longrightarrow \frac{\left[q_{2}\left(q_{1}+q_{2}\right)+z_{1}\left(z_{1}-z_{2}\right)\right] \sqrt{M^{2}+\ell^{2}}}{\left(q_{1}+q_{2}\right)^{2}+\left(z_{1}-z_{2}\right)^{2}} .
\end{aligned}
$$

These are the natural multi source generalisation of the parameters rescaling we performed in appendix B to get the Kerr-Newman metric in Boyer-Lindquist coordinates, as it can be seen comparing (B.8) with (5.14) and (B.7) with

$$
m_{1}+m_{2} \longrightarrow \sqrt{M^{2}+\ell^{2}} .
$$

Thanks to the above parameters redefinitions the complex potentials of the double Reissner-Nordstrom-NUT configuration takes the following form at spacial infinity, for $r \approx \sqrt{\rho^{2}+z^{2}} \rightarrow \infty$

$$
\begin{aligned}
& \mathcal{E} \sim 1-\frac{2(M-i \ell)}{r}+\frac{\text { const. }}{r^{2}}+O\left(\frac{1}{r^{3}}\right), \\
& \mathbf{\Phi} \sim \frac{2\left(q_{1}+q_{2}\right)}{r}+\frac{\left(q_{1} z_{1}+q_{2} z_{2}\right)\left[\left(q_{1}+q_{2}\right)^{2}+\left(z_{1}-z_{2}\right)^{2}-M^{2}-\ell^{2}\right] y}{\left[\left(q_{1}+q_{2}\right)^{2}+\left(z_{1}-z_{2}\right)^{2}\right] r^{2}}+\frac{\text { const. }}{r^{2}}+O\left(\frac{1}{r^{3}}\right) .
\end{aligned}
$$


Comparing eqs. (5.17)-(5.18) with the standard asymptotic potential decaying for a generic asymptotically flat solution, as given in (4.11)-(4.12) we can estimate the values of several physical quantities of the from the first terms of the multipolar expansion of the Ernst field of the double Reissner-Nordstrom-NUT space-time. In particular the total mass of the gravitational system is given by $M=\sqrt{\left(m_{1}+m_{2}\right)^{2}-\ell^{2}}$, the total electric charge of the space-time is $Q_{e}=q_{1}+q_{2}$, the NUT charge is $\ell$, the magnetic charge $Q_{m}$ and magnetic dipole moment $D_{m}$ are null. From the asymptotic fall-off (5.17)-(5.18) it can be read that the generated solution does not acquire any amount of angular momentum after the enhanced Ehlers transformation, i.e. $J=0$, even though it leaves its static state becoming stationary. So, in this case, the stationariness cannot be appreciated from the angular dipole, which being proportional to the angular momentum remains null. But the new parameter introduced by the Ehlers transformation can be appreciated from the contribution to others multipoles, such as the angular monopole or the angular quadrupole. In this sense the resulting spacetime is still twisting.

All that is in complete analogy with the well known examples of the Kerr-NUT spacetime, where the angular momentum is not affected by the value of the NUT parameter, which, contributes only from the following orders in the angular multipolar expansion. To clarify this point in the appendix B the firsts angular Geroch-Hansen multipole moments ${ }^{15}$ for the Kerr-NUT metric are computed.

Finally, from (4.12) and (5.18), we can also deduce the electric dipole of the above solution

$$
D_{e}=\frac{\left(q_{1} z_{1}+q_{2} z_{2}\right)\left[\left(q_{1}+q_{2}\right)^{2}+\left(z_{1}-z_{2}\right)^{2}-M^{2}-\ell^{2}\right]}{\left(q_{1}+q_{2}\right)^{2}+\left(z_{1}-z_{2}\right)^{2}} .
$$

In order to check the regularity of the solution on the axis of symmetry is sufficient to verify wether $\lim _{\rho \rightarrow 0} \gamma(\rho, z)=0$. However, as already noted in section 3, the Ehlers transformation applied to the seed metric of the form $(2.4)^{16}$ is not affecting $\gamma(\rho, z)$. Therefore the equilibrium condition $m_{1} m_{2}=q_{1} q_{2}$ might not be modified by the presence of the NUT parameter. Anyway, taking into account the case of the Kerr-Newman black hole, where the NUT charge produces not removable singularities, a more detailed study of this solution is due. In particular, since in this case the metric become stationary too, it is necessary to verify also the regularity on the symmetry axis of the rotational function $\omega(\rho, z)$, which turns not-null after applying the Ehlers transformation: $\lim _{\rho \rightarrow 0} \omega(\rho, z)=$ const. However this is outside the scope of the paper, but may be addressed in a future publication; for the rest of the article we will prefer to deal with a simpler case.

\subsection{The extremal case: Majumdar-Papapetrou-NUT spacetime}

Some stationary generalisations of the Majumdar-Papapetrou solution were firstly discovered by Israel and Wilson [23] and indipendently by Perjes [24] (IWP), mainly focusing in

\footnotetext{
${ }^{15}$ These are the same of the Beig-Simon multipole moments, while are equivalent to the Thorne ones only up to a constant factor.

${ }^{16}$ When applied to other variants of the LWP metric, for instance a double Wick rotated version of (2.4), which is used, for instance, to obtain black holes in external magnetic field, such as [27], the condition on $\gamma(\rho, z)$ is generally not sufficient.
} 
a multi Kerr-Newman sources. ${ }^{17}$ These proposals fall in a restricted class where the electromagnetic and gravitational Ernst potentials have a very specific linear relation between themself

$$
\boldsymbol{\Phi}=\mathfrak{a}+\mathfrak{b} \mathcal{E}, \quad \text { where } \quad \mathfrak{a b}^{*}+\mathfrak{a}^{*} \mathfrak{b}=-\frac{1}{2},
$$

for arbitrary complex parameters $\mathfrak{a}$ and $\mathfrak{b}$. These assumptions entails a constrained value between the masses and the electric charges of the constituents, which implies extremality only in the absence of the usual angular momentum parameter (often called $a$ ), otherwise the horizons turn out hyper-extremal.

Hawking and Hartle [25] have shown that the only multi sources solutions of these kind, without naked singularities, were the static and extremal ones discovered by Majumdar and Papapetrou. Note that the NUT generalisation of the multi sources non-extremal solution of section 5.1 does not belong to the family studied by Israel and Wilson. This can be done checking that the Ernst potentials (5.13) cannot be cast into (5.19); therefore the Hartle and Hawking no-go theorem does not apply for non-extremal metrics.

Hence the extremal case is of special interest between the whole family of two equilibrium Reissner-Nordstrom configurations [16, 18], because, only in this specific eventuality, both sources can be considered as regular black holes. For this reason it represents a good choice as a seed solution to analyse our generating technique in more detail. Also the concise and manifest form of the resulting metric, uncommon for stationary multiple gravitational sources, makes apparent the study of some thermodynamic properties for a configuration of double black holes.

For simplicity we choose to deal with an electrically charged seed only (i.e. the standard MP solution).

At extremality $m_{i}=e_{i}, \bar{\gamma}=\gamma=0=\sigma_{i}$, so the bipolar coordinates simplifies in

$$
x_{i}(\rho, z)= \pm \sqrt{\rho^{2}+\left(z-z_{i}\right)^{2}}, \quad y_{i}(\rho, z)= \pm \frac{z-z_{i}}{\sqrt{\rho^{2}+\left(z-z_{i}\right)^{2}}} .
$$

From eqs. (5.1)-(5.4) it is easy to find that the only non-trivial function of the LWP ansatz becomes

$$
f(\rho, z)=\left(1+\frac{m_{1}}{x_{1}}+\frac{m_{2}}{x_{2}}\right)^{-2}=\left[1+\frac{m_{1}}{\sqrt{\rho^{2}+\left(z-z_{1}\right)^{2}}}+\frac{m_{2}}{\sqrt{\rho^{2}+\left(z-z_{2}\right)^{2}}}\right]^{-2},
$$

while the electromagnetic potential, after a trivial gauge transformation given by a unitary constant shift with respect to (5.11), can be written as

$$
A_{\mu}=\left[-\left(1+\frac{m_{1}}{x_{1}}+\frac{m_{2}}{x_{2}}\right), 0,0,0\right] .
$$

Considering that the two sources are located symmetrically with respect to the origin of the $z$-axis, we can set, without losing generality, that $z_{1}=-\lambda$ and $z_{2}=\lambda$. In that case

\footnotetext{
${ }^{17}$ Also some others multi sources NUT metrics have been presented in [20], but without the electromagnetic field, thus these sources cannot sustain some equilibrium configuration.
} 
the only non-trivial function of the LWP ansatz, describing the Majumdar-Papapetrou solution, takes the economical form

$$
f=\lambda^{4} \frac{\left(x^{2}-y^{2}\right)^{2}}{E_{-}}=\left[1+\frac{m_{1}}{\lambda} \frac{1}{x+y}+\frac{m_{2}}{\lambda} \frac{1}{x-y}\right]^{-2}
$$

where

$$
\begin{aligned}
E_{ \pm} & =\lambda^{2}\left(x^{2}-y^{2}\right) \mp \lambda x\left(m_{1}+m_{2}\right) \pm \lambda y\left(m_{1}-m_{2}\right) \\
F & =\lambda\left[x\left(m_{1}+m_{2}\right)-y\left(m_{1}-m_{2}\right)\right]
\end{aligned}
$$

and the two-dimensional coordinates $(x, y)$ can be obtained as the inverse of the usual cylindrical coordinates (of the LWP ansatz)

$$
\left\{\begin{array}{l}
\rho=\lambda \sqrt{x^{2}-1} \sqrt{1-y^{2}} \\
z=\lambda x y
\end{array}\right.
$$

as follows ${ }^{18}$

$$
\left\{\begin{array}{l}
x=\frac{1}{2 \lambda}\left[\sqrt{\rho^{2}+(z+\lambda)^{2}}+\sqrt{\rho^{2}+(z-\lambda)^{2}}\right] \\
y=\frac{1}{2 \lambda}\left[\sqrt{\rho^{2}+(z+\lambda)^{2}}-\sqrt{\rho^{2}+(z-\lambda)^{2}}\right]
\end{array} .\right.
$$

In these coordinates the LWP metric (2.4) takes the form

$$
d s^{2}=-f(x, y)[d t+\omega(x, y) d \varphi]^{2}+\frac{\lambda^{2}}{f(x, y)}\left[\left(x^{2}-y^{2}\right) e^{2 \gamma(x, y)}\left(\frac{d x^{2}}{1-x^{2}}+\frac{d y^{2}}{y^{2}-1}\right)+\left(1-x^{2}\right)\left(y^{2}-1\right) d \varphi^{2}\right] .
$$

Thanks to the coordinates transformation (5.27) it is easy to see that the $f$ functions in eqs. (5.21) and (5.23) coincide. Moreover the electric field of (5.11) can be cast as $A_{t}=F / E_{-}$, while the remaining seed structure functions of the LWP metric are $\omega(x, y)=0$ and $\gamma(x, y)=0$. Hence the seed Ernst potentials for the Majumdar-Papapetrou solution are

$$
\mathcal{E}_{\mathrm{MP}}=\frac{E_{+}}{F}, \quad \quad \boldsymbol{\Phi}_{\mathrm{MP}}=A_{t}=\frac{F}{E_{-}}
$$

Applying the transformation (4.9) to the above seed we can add to the complex potentials the NUT parameter, as follow

$$
\mathcal{E}_{\mathrm{MPN}}=\frac{E_{+}+i b F}{F+i b E_{+}}, \quad \boldsymbol{\Phi}_{\mathrm{MPN}}=\frac{F^{2}(1+i b)}{E_{-}\left(F+i b E_{+}\right)}
$$

\footnotetext{
${ }^{18}$ More details about this transformation and the differential operators associated to the new set of coordinates can be found in appendix A.
} 
hence, taking into account the definitions (2.9), (2.10) and (2.11), we get ${ }^{19}$

$$
\begin{aligned}
f^{-1}(x, y) & =\left[\frac{m_{1}}{\lambda(x+y)}+\frac{m_{2}}{\lambda(x-y)}+\frac{1-b^{2}}{1+b^{2}}\right]^{2}+\left(\frac{2 b}{1+b^{2}}\right)^{2} \\
\omega(x, y) & =\frac{4 b}{1+b^{2}}\left[\frac{m_{2}(1-x y)}{x-y}-\frac{m_{1}(1+x y)}{x+y}\right]+\omega_{0} \\
A_{t}(x, y) & =\frac{\left[m_{1}(x-y)+m_{2}(x+y)\right]\left\{\frac{1-b^{2}}{1+b^{2}}\left[m_{1}(x-y)+m_{2}(x+y)\right]+\lambda\left(x^{2}-y^{2}\right)\right\}}{\left\{\left[m_{1}(x-y)+m_{2}(x+y)\right]+\frac{1-b^{2}}{1+b^{2}} \lambda\left(x^{2}-y^{2}\right)\right\}^{2}+\left[\frac{2 b \lambda}{1+b^{2}}\left(x^{2}-y^{2}\right)\right]^{2}} \\
A_{\varphi}(x, y) & =\omega(x, y) A_{t}(x, y)+A_{t 0} .
\end{aligned}
$$

As explained in section 3 the NUT transformation is not changing the decoupled function $\gamma(x, y)$, which remains null as the seed.

It can be easily checked that the Ernst potentials in (5.29) still belongs to the IWP class of solutions, as defined by (5.19), with

$$
\mathfrak{a}=-\mathfrak{b}=\frac{i-b}{2(i+b)} .
$$

To better understand the physical properties of the solution the polar coordinates centred in one of the two black hole, let's say the black hole of mass $m_{1}$,

$$
\begin{aligned}
& \bar{r}=\sqrt{\rho^{2}+(z+\lambda)^{2}}, \\
& \theta=\arctan \left(\frac{\rho}{z+\lambda}\right),
\end{aligned}
$$

are useful. $\bar{r}=r-m_{1}$ and the $\theta$ axis is aligned with the two mass points. In these coordinates the Majumdar-Papapetrou-NUT metric functions $f$ and $\omega$ of the LWP metric (2.4)

$$
d s^{2}=-f(\bar{r}, \theta)[d t+\omega(\bar{r}, \theta) d \phi]^{2}+f^{-1}(\bar{r}, \theta)\left[d \bar{r}^{2}+\bar{r}^{2} d \theta^{2}+\bar{r}^{2} \sin ^{2} \theta d \varphi^{2}\right],
$$

read respectively

$$
\begin{aligned}
f(\bar{r}, \theta)^{-1} & =\left(\frac{1-b^{2}}{1+b^{2}}+\frac{m_{1}}{\bar{r}}+\frac{m_{2}}{\sqrt{\bar{r}^{2}-4 \lambda \bar{r} \cos \theta+4 \lambda^{2}}}\right)^{2}+\left(\frac{2 b}{1+b^{2}}\right)^{2}, \\
\omega(\bar{r}, \theta) & =\frac{4 b}{1+b^{2}}\left[-m_{1} \cos \theta+m_{2}\left(\frac{2 \lambda-\bar{r} \cos \theta}{\sqrt{\bar{r}^{2}-4 \lambda \bar{r} \cos \theta+4 \lambda^{2}}}\right)\right] .
\end{aligned}
$$

While also the associated electromagnetic field in coordinates $(\bar{r}, \theta)$ can be easily found from eqs. (5.32) and (5.33) by a coordinates transformation that can be obtained by combining the two transformations (5.27) and (5.35)-(5.36), as shown in appendix A. In these

\footnotetext{
${ }^{19}$ A Mathematica notebook with the Majumdar-Papapetrou-NUT solution, in several coordinates, can be found at https://sites.google.com/site/marcoastorino/papers/majumdar-papapetrou-nut-black-hole.
} 
coordinates is very clear that the physical boundary conditions for the asymptotic decaying of the Ernst potential additionally imposed by Israel and Wilson,

$$
\mathcal{E} \underset{\bar{r} \rightarrow \infty}{\longrightarrow} 1, \quad \boldsymbol{\Phi} \underset{\bar{r} \rightarrow \infty}{\longrightarrow} 0,
$$

are automatically implemented by the enhanced Ehlers transformation. On the other hand this is not true for the standard Ehlers transformation, where the gravitational Ernst potential $\mathcal{E}$ goes asymptotically to a complex constant, when applied to the MajumdarPapapetrou solution.

The limits to the well known solution, such as the extremal Reissner-Nordstrom or the static Majumdar-Papepetrou are very clear. To get the extremal Reissner-Nordstrom it is sufficient to vanish the second black hole, through the $m_{2} \rightarrow 0$ limit, while the Majumdar-Papepetrou, in the standard coordinates presented in $[25,26]$ is retrieved when the nut parameter $b$ is null (the relation between the nut parameter $b$ of the enhanced and generalised Reina-Treves transformation is related to the standard NUT parameter as seen in eq. (B.8)).

The two event horizons are located at $\rho=0$ and $z= \pm \lambda$, thus their distance is $2 \lambda$. Actually, in terms of the radial coordinate centred in the $m_{1}$ black hole, the loci identified by $\rho=0$ and $z=-\lambda$ correspond to $\bar{r}=0$, which is not a point but it represents a surface of finite area

$$
\left.\mathcal{A}_{1}\right|_{\bar{r}=0}=\left.\int_{0}^{2 \pi} d \varphi \int_{0}^{\pi} d \theta \sqrt{g_{\theta \theta} g_{\varphi \varphi}}\right|_{\bar{r}=0}=4 \pi m_{1}^{2} .
$$

Moreover from the inspection of the polar circumference (meridian)

$$
C_{p}=\left.\int_{0}^{2 \pi} \sqrt{g_{\theta \theta}} d \theta\right|_{\bar{r}=0}=2 \pi m_{1}
$$

and azimuthal circumference (parallels)

$$
C_{a}=\left.\int_{0}^{2 \pi} \sqrt{g_{\varphi \varphi}} d \varphi\right|_{\bar{r}=0}=2 \pi m_{1} \sin \theta
$$

of the event horizon of mass $m_{1}$ (at constant $t$ ) it is possible to infer the spherical symmetry of the event horizon.

Specular result for the event horizon area and circumferences can be obtained using a set of coordinates centred in the second black hole, just replace $m_{1}$ with $m_{2}$.

Following the reasoning of Hartle and Hawking in [25] for the NUT free case, it is possible to show that the geometry at $\bar{r}=0$ is regular and it describes a null surface. At this scope consider the following coordinates transformation

$$
t=u+F(\bar{r})
$$

where the $F(\bar{r})$ function is chosen such that

$$
\frac{d F}{d \bar{r}}=\left[\left.\sqrt{f^{-1}(\bar{r}, \theta)}\right|_{r \approx 0}\right]^{2}=\left[\frac{m_{1}}{\bar{r}}+\frac{m_{2}\left(1+b^{2}\right)+2 \lambda\left(1-b^{2}\right)}{2 \lambda\left(1+b^{2}\right)}\right]^{2}=: V^{2}(\bar{r}) .
$$


Then, defining $\mathrm{U}(r):=\sqrt{f^{-1}(\bar{r}, \theta)}$, the metric becomes

$$
\begin{aligned}
d s^{2}= & -\frac{d u^{2}}{U^{2}(\bar{r})}+d \bar{r}^{2}\left[\frac{U^{4}(\bar{r})-V^{4}(\bar{r})}{U^{2}(\bar{r})}\right]-2 \frac{V^{2}(\bar{r})}{U^{2}(\bar{r})} d u d \bar{r}+2 \frac{\omega(\bar{r}, \theta)}{U^{2}(\bar{r})} d u d \varphi+2 \frac{V^{2}(\bar{r})}{U^{2}(\bar{r})} d \bar{r} d \varphi \\
& +\frac{\omega^{2}(\bar{r}, \theta)}{U^{2}(\bar{r})} d \varphi^{2}+U^{2}(\bar{r}) \bar{r}^{2}\left(d \theta^{2}+\sin ^{2} \theta d \varphi^{2}\right),
\end{aligned}
$$

which, in the neighbourhood of $\bar{r}=0$, it simplifies to

$$
\begin{aligned}
d s^{2}= & -\frac{\bar{r}^{2}}{m_{1}^{2}} d u^{2}+\left[\frac{8 b^{2} \lambda^{2}+\left(1+b^{2}\right)^{2} m_{1} m_{2} \cos \theta}{1+b^{2}}\right] d \bar{r}^{2}-2 d u d \bar{r} \\
& +\left[\frac{m_{2}+m_{1} \cos \theta}{1+b^{2}}\right] 8 b\left[\frac{\bar{r}^{2}}{m_{1}^{2}} d u d \varphi+d \bar{r} d \varphi+2 b \frac{\bar{r}^{2}}{m_{1}^{2}} d \varphi^{2}\right]+m_{1}^{2}\left(d \theta^{2}+\sin ^{2} \theta d \varphi^{2}\right) .
\end{aligned}
$$

The above metric is explicitly regular at $\bar{r}=0$, thus we can extend the manifold to negative values of $\bar{r}$, furthermore it's clear that $\bar{r}=0$ describes a null-surface. Thanks to the following definitions

$$
r_{1}=-\bar{r}, \quad r_{2}=\sqrt{r_{1}^{2}+4 \lambda r_{1} \cos \theta+4 \lambda^{2}}
$$

the extended spacetime is described by the metric (5.37) but with $f(\bar{r}, \theta)$ replaced by

$$
\tilde{f}\left(r_{1}, \theta\right)^{-1}:=\left(\frac{1-b^{2}}{1+b^{2}}-\frac{m_{1}}{r_{1}}+\frac{m_{2}}{r_{2}}\right)^{2}+\left(\frac{2 b}{1+b^{2}}\right)^{2} .
$$

In the known case of standard Majumdar-Papapetrou metric, for $b=0$, let's call the content of the non-vanishing bracket of $(5.48) \tilde{U}_{0}\left(r_{1}, \theta\right)$. In that case the function $\tilde{U}_{0}\left(r_{1}, \theta\right)$, close to $r_{1}=0$ takes large negative values, while for $r_{2} \approx 0$ takes positive values, so it must vanish somewhere in between, where the metric diverges. Actually from the curvature scalar invariants, ${ }^{20}$ it is possible to realise that the zeros of $\tilde{U}_{0}$ are points where the curvature invariants diverge, while as we have seen above, $r_{1}=0$ it is just a coordinate singularity. The locus of $\tilde{U}_{0}\left(r_{1}, \theta\right)=0$ is not a surface but a point. It can be shown, just considering a surface inside the domain defined by $\tilde{U}_{0}\left(r_{1}, \theta\right)=0$ and letting it tend to $\tilde{U}_{0}\left(r_{1}, \theta\right)=0$, we get

$$
\int_{0}^{2 \pi} d \varphi \int_{0}^{\pi} d \theta \tilde{U}_{0}^{2}\left(r_{1}, \theta\right) r_{1}^{2} \sin \theta \quad \longrightarrow 0
$$

So, for $b=0$, the region interior to $r_{1}=0$ shows similarities with the interior of the RN black hole.

When $b \neq 0$ the function $\tilde{f}^{-1}\left(r_{1}, \theta\right)$ cannot vanish therefore the metric, thanks to the nut parameter $b$ becomes regular, exactly as occurs in the case of a single NUT black hole, where the curvature singularity is smeared by the NUT parameter. Therefore the radial coordinate $\bar{r}$ can be continued without obstructions for negative values. This feature recently makes people think that black holes generalisation with the NUT parameter are suitable, under certain assumptions, to traversable wormhole interpretation ${ }^{21}$ [30].

\footnotetext{
${ }^{20}$ The curvature scalar invariants are computationally easier to obtain in the $(x, y)$ coordinates.

${ }^{21}$ While some constraints on the upper bound of the NUT parameter were proposed, the presence of closed-timelike curves, that violate causality, for free-falling observers cannot completerly discarted in [30].
} 


\subsection{Warm up: Reissner-Nordstrom-NUT/CFT correspondence}

The Kerr-CFT correspondence aims to describe the microscopical degrees of freedom of a black hole, especially at extremality, through a duality between the near horizon geometry of the black hole and a conformal field theory dual to the near horizon geometry placed on its asymptotic region, [33] and [34].

It would be interesting to check if the tools provided by the Kerr-CFT can be extended in the context of multi black holes.

From the extremal near horizon geometry of the Majumdar-Papapetrou-NUT black hole it is possible to extract the central charge of the dual CFT associated to the black hole solution. Unfortunately in the case of extremal charged black hole with NUT the diagonal form of the near horizon metric makes difficult to implement the standard stationary KerrCFT picture, where the central charge is strongly related to the rotation of the black hole.

On the other hand, for the electrically charged black hole solutions, it is possible to exploit an alternative approach which is not based on the rotational symmetry around the azimuthal angle, but on the U(1) symmetry of the Maxwell gauge potential. The KaluzaKlein uplift of our solution in one dimension more transforms the electromagnetic degrees of freedom in rotational degrees of freedom, as explained in [34] (see also [36] and [37]). In fact, considering the Abelian gauge field to be wrapped around a compact extra dimension $\psi$ of period $2 \pi R_{\psi}$, we can define an extremal Frolov-Thorne vacuum, which corresponds to a temperature in the boundary conformal field theory, ${ }^{22}$ in units of $R_{\psi}: T_{\psi}=T_{e} R_{\psi}$. In analogy with the standard rotational picture, at extremality, the electromagnetic chemical potential $T_{e}$ can be defined as

$$
T_{e}:=\lim _{r_{+} \rightarrow r_{+}^{\text {ext }}} \frac{T_{\mathrm{H}}}{\Psi_{e}^{\mathrm{ext}}-\Psi_{e}},
$$

where the $\Psi_{e}$ represents the Coulomb electromagnetic potential evaluated at the event horizon ( $\Psi_{e}^{\text {ext }}$ is the extremal case), while $T_{\mathrm{H}}$ is the usual Hawking temperature.

To clarify some points let's review some aspects of the microscopic entropy the Reissner-Nordstrom-NUT black holes, recently studied also in [38]. The line element and the gauge vector potential of the dyonic generalisation of the solution found by Brill in [35] are given respectively by

$$
d s^{2}=-\left(\frac{r^{2}-2 m r+q^{2}+p^{2}-\ell^{2}}{r^{2}+\ell^{2}}\right)[d t+2 \ell \cos \theta d \varphi]^{2}+\frac{d r^{2}}{\frac{r^{2}-2 m r+q^{2}+p^{2}-\ell^{2}}{r^{2}+\ell^{2}}}+\left(r^{2}+\ell^{2}\right)\left[d \theta^{2}+\sin ^{2} \theta d \varphi^{2}\right]
$$

and

$$
A_{\mu}=\left[-\frac{q r+p \ell}{r^{2}+\ell^{2}}, 0,0, \frac{p\left(r^{2}-\ell^{2}\right)-2 q r \ell}{r^{2}+\ell^{2}} \cos \theta\right] .
$$

The metric presents two Killing horizons, an inner $r_{-}$and outer $r_{+}$one, located at $r_{ \pm}=$ $m \pm \sqrt{m^{2}-q^{2}-p^{2}+\ell^{2}}$. In the extremal case they coincide at $r_{+}^{\text {ext }}=\sqrt{q^{2}+p^{2}-\ell^{2}}$.

\footnotetext{
${ }^{22}$ Since on the dual CFT model the Frolov-Thorne vacuum plays the role of a temperature it is often called Frolov-Thorne temperature.
} 
Since the angular velocity at the event horizon is null, $\Omega_{J}=0$, the Killing vector generating the event horizon remains the same of the Reissner-Nordstrom one: $\chi=\partial_{t}$. Then the Hawking temperature $T_{\mathrm{H}}$ is defined from the surface gravity $\kappa_{s}$ as follows

$$
T_{\mathrm{H}}=\frac{\kappa_{s}}{2 \pi}=\left.\frac{1}{2 \pi} \sqrt{-\frac{1}{2} \nabla_{\mu} \chi_{\nu} \nabla^{\mu} \chi^{\nu}}\right|_{r_{+}}=\frac{1}{4 \pi} \frac{r_{+}-r_{-}}{r_{+}^{2}+\ell^{2}} .
$$

Thus at extremality $\left(r_{+}=r_{-}\right)$the Hawking temperature become null. Nevertheless the electromagnetic chemical potential, as defined in (5.50), is well defined and non null for the extreme RN-NUT black hole

$$
{ }^{[\mathrm{RNN}]} T_{e}=\frac{1}{2 \pi} \frac{p^{2}+q^{2}}{p^{2} q+q^{3}-2 q \ell^{2}+2 p \ell \sqrt{p^{2}+q^{2}-\ell^{2}}},
$$

since the Coulomb electromagnetic potential for RN spacetime is given by

$$
{ }^{[\mathrm{RNN}]} \Psi_{e}=-\left.\chi^{\mu} A_{\mu}\right|_{r_{+}}=\frac{q r_{+}+p \ell}{r_{+}^{2}+\ell^{2}} .
$$

To reach the extremal RN-NUT near horizon metric the following change of coordinates adapted to the horizon is needed

$$
r(R):=r_{+}^{\mathrm{ext}}+\beta r_{0} R, \quad t(\tau):=\frac{r_{0}}{\beta} \tau, \quad \varphi(\tau, \phi):=\phi+\Omega_{J} \frac{r_{0}}{\beta} \tau .
$$

Taking the limit for $\beta \rightarrow 0$ the metric, near the horizon of an extreme RN-NUT black hole, takes the form of warped product of $A d S_{2} \times S^{2}$. In particular, as shown in [39] or [34], it can be cast into the standard form of an extremal near-horizon geometry

$$
d s^{2}=\Gamma(\theta)\left[-R^{2} d \tau^{2}+\frac{d R^{2}}{R^{2}}+\alpha^{2}(\theta) d^{2} \theta+\gamma^{2}(\theta)(d \phi+\kappa R d \tau)^{2}\right]
$$

for

$$
\Gamma(\theta)=q^{2}+p^{2}, \quad \alpha(\theta)=1, \quad \kappa=0, \quad \gamma(\theta)=\sin \theta .
$$

To write the near horizon gauge vector potential, in addition to the coordinates transformation (5.56), the electric potential have to be gauge shifted: $A_{t} \longrightarrow A_{t}+\Psi_{e}$. Then in the near-horizon limit, for $\beta \rightarrow 0$ we have

$$
A_{\mu}=\left[R \frac{p^{2} q+q^{3}-2 q \ell^{2}+2 p \ell \sqrt{p^{2}+q^{2}-\ell^{2}}}{p^{2}+q^{2}}, 0,0, \cos \theta \frac{q^{2} p+p^{3}-2 p \ell^{2}-2 q \ell \sqrt{p^{2}+q^{2}-\ell^{2}}}{p^{2}+q^{2}}\right] .
$$

Comparing the above potential with the general near horizon form for the static case,

$$
A=\bar{e} R d \tau-\frac{\bar{p}}{4 \pi} \cos \theta d \phi,
$$

we can extract the value of the constant

$$
\bar{e}=\frac{p^{2} q+q^{3}-2 q \ell^{2}+2 p \ell \sqrt{p^{2}+q^{2}-\ell^{2}}}{p^{2}+q^{2}} .
$$


Hence the electromagnetic chemical potential for the RN-NUT black hole can be written as

$$
\left.{ }^{[\mathrm{RNN}}\right]_{e}=\frac{1}{2 \pi \bar{e}} .
$$

Finally the central charge of the dual conformal field model living on the boundary of the near horizon metric, as explained in $[34,37]$ is given by

$$
c_{Q}=\frac{3 \bar{e}}{R_{\psi}} \int_{0}^{\pi} \Gamma(\theta) \gamma(\theta) \alpha(\theta) d \theta=\frac{6 \bar{e}}{R_{\psi}}\left(q^{2}+p^{2}\right) .
$$

According to the Kerr/CFT correspondence, the microscopic entropy of the extremal RNNUT black hole can be obtained by the Cardy formula, considering as the left central charge the $c_{Q}$ and as the left temperature ${ }^{23} T_{\psi}$

$$
\mathcal{S}_{\mathrm{CFT}}=\frac{\pi^{2}}{3} c_{L} T_{L}=\pi\left(q^{2}+p^{2}\right)=\frac{\mathcal{A}}{4} .
$$

So we get that the microscopic entropy of the extremal Reissner-Nordstrom-NUT coincides with a quarter of its horizon area $\mathcal{A}$

$$
\mathcal{A}=\int_{0}^{2 \pi} d \varphi \int_{0}^{\pi} d \theta \sqrt{g_{\theta \theta} g_{\varphi \varphi}}=\left.4 \pi\left(r_{+}^{2}+\ell^{2}\right)\right|_{r_{+} \rightarrow r_{+}^{\text {ext }}}=4 \pi\left(q^{2}+p^{2}\right) .
$$

Note that, even though the presence of the NUT parameter modifies the position of the horizons, it is not affecting the final result: the entropy remain the same of the standard dyonic extreme Reissner-Nordstrom black hole. Note also that the microscopic entropy of RNN black hole, according to the Kerr/CFT correspondence agrees with the BekensteinHawking law, but it does not agree with others entropy proposals, such as [46].

\subsection{Near horizon geometry of the Majumdar-Papapetrou-(NUT) spacetime}

As we have seen in section 5.3, for a single extremal charged black hole it is known that the near-horizon geometry is not affected directly by the presence of the NUT parameter. It would be interesting to see if the same result holds also for the double extremal charged black hole endowed with the NUT charge. At this scope let's consider the di-hole solution in the coordinates centred in one of the black holes, given by the eqs. (5.37)-(5.39). To get the near horizon limit it is propaedeutic a change of coordinates adapted (and in a co-rotating frame with respect) to the event horizon

$$
\bar{r}(R):=\bar{r}_{+}+\beta r_{0} R, \quad t(\tau):=\frac{r_{0}}{\beta} \tau, \quad \varphi(\tau, \phi):=\phi+\Omega_{J} \frac{r_{0}}{\beta} \tau .
$$

The constant $r_{0}$ is needed to remain with dimensionless coordinates, while $\Omega_{J}$ is the angular velocity at the horizon $r_{+}$, which results null even though the metric is stationary:

$$
\Omega_{J}:=-\left.\frac{g_{\theta \phi}}{g_{\phi \phi}}\right|_{r_{+}}=0
$$

\footnotetext{
${ }^{23}$ The right temperature is null for extreme configuration.
} 
As explained above, in these coordinates the event horizon is where $\bar{r}$ vanishes, i.e. $\bar{r}_{+}=$ 0 . Taking the limit for $\beta \rightarrow 0$, and choosing $r_{0}=m_{1}$, we obtain the geometry of the Majumdar-Papapetrou-NUT spacetime near the horizon of the black hole of mass $m_{1}$

$$
\tilde{d s}^{2}=m_{1}\left[-R^{2} d \tau^{2}+\frac{d R^{2}}{R^{2}}+d \theta^{2}+\sin ^{2} \theta d \phi^{2}\right] .
$$

It's worth to point out that this near horizon geometry coincides exactly with the one of the extreme Reissner-Nordstrom-(NUT) black hole and that it does not depends on the NUT parameter, as in the single black hole case. Also note that in the presence of an ensemble of black holes, the geometry near the event horizon of a single black hole still falls in the standard class of the extremal near horizon geometries described in [39]. In the case under consideration, we can infer, from eq. (5.67), that the near horizon metric represents a warped product of $A d S_{2} \times S^{2}$, thus it is endowed with the $\mathrm{SL}(2, \mathbb{R}) \times \mathrm{U}(1)$ isometry.

Of course a similar result can be obtained using coordinates centred in the black hole of mass $m_{2}$, just replacing $m_{1}$ with $m_{2}$. Actually from the Majumdar-PapapetrouNUT solution written in coordinates $(\rho, z),{ }^{24}$ it is possible to simultaneously write both near horizon geometries, when the mass of the two black hole coincides, i.e. $m_{2}=m_{1}$, considering the near-horizon change of coordinates

$$
\rho(R, \theta):=\beta r_{0_{\mp}} R \sin \theta, \quad z(R, \theta):=\mp \lambda+\beta r_{0_{\mp}} R \cos \theta, \quad t(\tau):=\frac{r_{0_{ \pm}}}{\beta} \tau,
$$

and performing the $\beta \rightarrow 0$ limit. The resulting near horizon geometry is described by the metric

$$
\tilde{d s}^{2}=m_{i}^{2}\left[-R^{2} d \tau^{2}+\frac{d R^{2}}{R^{2}}+d \theta^{2}+\sin ^{2} \theta d \phi^{2}\right],
$$

where $i=1$ is associated to the source centred in $(\rho=0, z=-\lambda)$, while $i=2$ to the one localised by $(\rho=0, z=+\lambda)$. When the masses of the sources coincide $\left(m_{1}=m_{2}\right)$, then $r_{0_{-}}=r_{0_{+}}=r_{0}=m_{1}$, when the masses do not coincide $r_{0_{-}}=m_{1}$ and $r_{0_{+}}=m_{2}$. As always occurs for regular extremal black hole this metric models an $A d S \times S^{2}$ spacetime.

To obtain the near horizon form of the electromagnetic potential we firstly perform the usual gauge transformation on the vector potential $A_{t} \rightarrow A_{t}+\Psi_{e}$ of the non-extremal solution (5.11); then in the coordinates adapted to the horizon we take the $\beta \rightarrow 0$ limit, which gives

$$
A=\bar{e} R d \tau, \quad \text { where } \quad \bar{e}=\frac{r_{0_{\mp}}^{2}}{m_{1}} .
$$

Following the methods provided by the Kerr/CFT framework [33, 34] it is possible to evaluate the, so called, microscopic entropy of the Majumdar-Papapetrou di-hole. In the case under consideration multiple disconnected event horizons are present therefore the procedure, originally found for a single black hole, have to be somehow adapted. The more

\footnotetext{
${ }^{24}$ See appendix C for details.
} 
straightful case is certainly when the two extremal sources have the same mass, because in that case the boundary condition of the near horizon geometry can be considered on the same footing. On the other hand, when the two sources are not identical, the easier approach is to treat each one individually. According to the standard prescription given by the Kerr/CFT correspondence, the microscopic entropy of the extremal black hole is associated to the (left) temperature and the central charge of the associated modular invariant conformal field theory model located on the boundary of the near horizon solution, can be derived by the Cardy formula ${ }^{25}$

$$
\mathcal{S}_{\mathrm{CFT}}=\frac{\pi^{2}}{3} c_{L} T_{L}
$$

As we have seen in the single black hole case studied in section 5.3, the left temperature of the boundary conformal field theory is associated with the chemical potential $T_{e}$, defined in (5.50) and (5.61). Because the Majumdar-Papapetrou di-hole is associated to a couple of conformal systems we treat the above quantities additively, as follows

$$
\begin{aligned}
c_{L} & =\sum_{i=1}^{2} \frac{3 \bar{e}_{i}}{R_{\psi}} \int_{0}^{\pi} \Gamma_{i}(\theta) \gamma(\theta) \alpha(\theta) d \theta=\frac{6 \bar{e}_{i}}{R_{\psi}} m_{i}^{2}, \\
T_{L} & =\sum_{i=1}^{2} R_{\psi} T_{e_{i}}=\sum_{i=1}^{2} \frac{R_{\psi}}{2 \pi \bar{e}_{i}} .
\end{aligned}
$$

In this way, from (5.71)-(5.73), the resulting entropy of the conformal field model associated to the double black hole ensemble described by Majumdar-Papapetrou solution is given by

$$
\mathcal{S}_{\mathrm{CFT}}=\sum_{i=1}^{2} \pi m_{i}^{2}=\pi\left(m_{1}^{2}+m_{2}^{2}\right)=\frac{\mathcal{A}_{1}+\mathcal{A}_{2}}{4}=\frac{\mathcal{A}}{4} .
$$

As expected, the entropy of the gravitational system, inferred by the conformal field techniques and the near horizon analysis, coincides with a quarter of the total area $\mathcal{A}$ of the event horizons of the two black holes, in agreement with the Bakenstein-Hawking formula.

Lastly we stress the utility of the non-extremal solution of section 5.1, in the above framework, because the chemical potential $T_{e}$ is defined through a limit from non-extremal quantities.

\subsection{Second law of thermodynamics}

Even though the solution we generated is not dynamical and therefore it can not describe the collision and merging process of the two sources, as time changes, we still can estimate which of the two configurations described either by the disjoint ensemble of two black holes of Majumdar-Papapetrou type or by the collapsed configuration, is thermodynamically favoured; specifically which of the two has the bigger entropy.

The collapsed configuration can be obtained just by letting the distance between the two sources, $l$, as defined in (5.9), going to zero, i.e. $\lambda \rightarrow 0$. In that case the resulting black

\footnotetext{
${ }^{25}$ For the details concerning the applicability of the Cardy formula we refer to the review [34].
} 
hole has the total mass and electric charge given by the sum of the ensemble components. Note that both configurations have the same total mass and total electric charge.

Extremal black hole entropy is still a puzzling issue even for a single source. In fact, while from the semiclassical action approach [47-49] the extremal black hole entropy seems to be null, from a microscopical point of view, according to string theory [50] or the Kerr/CFT correspondence [33], the black hole entropy follows the usual BekensteinHawking formula, i.e. a quarter of the event horizon area. Works in the licterature trying to clarify the discrepancy, such as [51] and [52], point to validate the Bekentein-Hawking area law also at extremality.

Black hole entropy becomes even a more puzzling issue when the NUT parameter deforms the extremal black hole, because there are different results, where the NUT parameter plays an active role or not, depending also on the interpretation of the Misner string related to these spacetimes, [53].

In section 5.3 it is shown how, according to the Kerr/CFT correspondence approach, for a single extremal and charged black hole with NUT the Bekenstein-Hawking formula holds (without any role played by the NUT parameter). Furthermore we have seen, in the previous subsection, as the same computation for the entropy of the double source solution can be also realised.

As the strong similitude between the near horizon geometry of the single and of the double extremal systems suggests, we have found that also the entropy of the MajumdarPapapetrou-(NUT) black holes is given by a quarter of its event horizon area.

Actually even without relying on the duality relation with the conformal field theory, just from the near-horizon metric $\tilde{d s}$, as in (5.69), it is very easy to deduce the areas $\mathcal{A}_{1}$ and $\mathcal{A}_{2}$ of each of the couple of black holes, which is

$$
\mathcal{A}_{i}=\int_{0}^{2 \pi} d \phi \int_{0}^{\pi} d \theta \sqrt{\tilde{g}_{\theta \theta} \tilde{g}_{\phi \phi}}=4 \pi m_{i}^{2} .
$$

Hence, assuming the validity of the Bekenstein-Hawking entropy (as shown above in the framework of the Kerr/CFT correspondence), the total entropy provided by the system of two separated extremal black holes $\mathcal{S}_{\odot \odot}$ come out to be proportional to the sum of the two horizon surfaces

$$
\mathcal{S}_{\odot \odot}=\frac{1}{4} \sum_{i=1}^{2} \mathcal{A}_{i}=\pi\left(m_{1}^{2}+m_{2}^{2}\right) .
$$

On the other hand the entropy $\mathcal{S}_{\odot}$ of the system where the two black holes are joint is given by

$$
\mathcal{S}_{\odot}=\frac{\mathcal{A}_{\odot}}{4}=\pi\left(m_{1}+m_{2}\right)^{2} .
$$

Therefore, for the same values mass and electric charge, the collapsed single black hole is the thermodynamically favoured configuration, due to its higher entropy

$$
\mathcal{S}_{\odot \odot} \leq \mathcal{S}_{\odot}
$$


in agreement with the Hawking area law, which states that from a classical point of view ${ }^{26}$ the even horizon area of a black hole tends to increase. Hence this relatively simple model allows us to confirm that the second law of black hole thermodynamics is verified for extremal (and charged) binary merging. A similar result have been found recently for a different couple of black holes held at equilibrium by a conical singularity [54].

Note that the equality in eq. (5.78) holds only if one of the two black holes disappears, which occurs when its mass parameter vanish; for all other proper double source configuration only the strict inequality holds.

In all this thermodynamics discussion the presence of the NUT parameter is quite irrelevant, as it does not directly plays a role in the final values of the charged black hole entropy (nor area), as it occurs also in the single source seen in section 5.3. Therefore we can state that, also for the standard double Majudar-Papapetrou di-hole spacetime, the more entropic state is given by the collapsed one.

\section{Summary, comments and conclusions}

In this article a detailed study of the Ehlers transformation is presented. It is shown, through explicit examples, why the known methods used to add the NUT parameter to an axisymmetric and stationary spacetime in general relativity are not precise when the Maxwell electromagnetic field is coupled to the gravitational theory.

We have shown how to modify the Ehlers and the Reina-Treves transformations in order to generalise an electrovacuum spacetime by adding the NUT parameter. The transformation is also able to remove the NUT charge when it is applied to a seed endowed with an undesired NUT parameter, without deforming the electromagnetic potential.

By analysing the Ernst potentials generated by the new transformation proposed we understand that it acts as a duality transformation between the mass and the gravitomagnetic charge, in a similar way the duality transformation for the Maxwell electromagnetism rotates the electromagnetic field. As a future perspective would be interesting to deepen this point and, in particular, to explore the possibility of finding other kinds of dualities for the gravitational theory, even outside of the axisymmetric and stationary setting.

We have applied this enhanced transformation of the Ernst equations to obtain a new solution representing a NUT generalisation of a couple of charged black holes at equilibrium. The extremal limit of this metric gives the Majumdar-Papapetrou-NUT spacetime.

Moreover thanks to the near horizon techniques some thermodynamic properties of the Majumdar-Papapetrou-(NUT) solution have been studied. Since the solution, when varying the parameters, can describe both a single or a double black hole event horizon, it is possible to confirm which configuration of the system is favoured from a thermodynamic point of view. The outcome is in agreement with the second law of black hole thermodynamics because, for a given mass, the bigger entropy configuration is obtained when the parameters of the solution are such that the two black hole are collapsed in a single one, which have a bigger area as well.

\footnotetext{
${ }^{26}$ With classical we mean we are ignoring possible quantum mechanical effects that might cause the evaporation of black hole, thought emission of Hawking radiation.
} 
The extremal character of the Majumdar-Papapetrou-(NUT) solution provide a good testing ground to prove the applicability of the techniques borrowed from the Kerr/CFT correspondence also for multi black hole configurations. In particular it was possible to reproduce the microscopic entropy for the extremal charged di-hole system.

We observe that, following [55]-[56], the procedure illustrate here is easily generalisable to the presence of a minimally or a conformally coupled scalar field ${ }^{27}$ (and all the related scalar-tensor gravitational theories connected with them by a conformal transformation, such as some Branse-Dicke or $f(R)$ gravities). On the other hand the generalisation to the presence of the cosmological constant is not that easy because the alleged breaking of the symmetries of the Ernst equations [41].

\section{Acknowledgments}

I would like to thank Vladimir Manko for fruitful discussions and for suggesting references. This work has been funded by Fondecyt project n\&\#9702;11160945, partially by the Conicyt - PAI grant n\&\#9702;79150061, by Beca Chile, INFN and by MIUR-PRIN contract 2017CC72MK-003.

\section{A Notation: differential operators in various coordinates}

Let's fix the notation of section 3, in particular the definition of differential operators in various coordinate systems. One of the advantages of writing the Einstein-Maxwell equations using the Lewis-Wayl-Papapetrou ansatz in coordinates $(\rho, z)$ is that the gravitational equations, which usually are written by curved differential operators, can be easier written in terms of flat differential operators. The same non-trivial property is inherited also by the complex Ernst equations.

For this reason we are interested in flat three-dimensional spacetime in cylindrical coordinates $(\rho, z, \varphi)$, whose metric can be written as

$$
d s^{2}=d \rho^{2}+d z^{2}+\rho^{2} d \varphi^{2} .
$$

For any scalar $f(\rho, z, \varphi)$ or vectorial function $\vec{A}(\rho, z, \varphi)$, the gradient, the divergence and the laplacian are respectively

$$
\begin{aligned}
\vec{\nabla} f(\rho, z, \varphi) & =\vec{e}_{\rho} \frac{\partial f(\rho, z, \varphi)}{\partial \rho}+\vec{e}_{z} \frac{\partial f(\rho, z, \varphi)}{\partial z}+\vec{e}_{\varphi} \frac{\partial f(\rho, z, \varphi)}{\partial \varphi} \\
\vec{\nabla} \cdot \vec{A}(\rho, z, \varphi) & =\frac{1}{\rho} \frac{\partial}{\partial \rho}\left(\rho A_{\rho}\right)+\frac{\partial A_{z}}{\partial z}+\frac{\partial A_{\varphi}}{\partial \varphi} \\
\nabla^{2} f(\rho, z, \varphi) & =\frac{\partial^{2} f}{\partial \rho^{2}}+\frac{\partial^{2} f}{\partial z^{2}}+\frac{1}{\rho^{2}} \frac{\partial^{2} f}{\partial \varphi^{2}}+\frac{1}{\rho} \frac{\partial f}{\partial \rho}
\end{aligned}
$$

Note that for the axisymmetric case under consideration in this paper (A.2)-(A.4) simplify further because no function depends on the $\varphi$ angle.

\footnotetext{
${ }^{27}$ In particular the NUT generalisation of the Bekenstein black hole is worked out in [57].
} 
The three dimensional cylindrical coordinates $(\rho, z, \varphi)$ are related to prolate spheroidal coordinates $(x, y, \zeta)$ with the following transformation

$$
\begin{aligned}
& \rho(x, y, \zeta)=\lambda \sqrt{x^{2}-1} \sqrt{1-y^{2}} \cos \zeta \\
& z(x, y, \zeta)=\lambda x y \\
& \varphi(x, y, \zeta)=\lambda \sqrt{x^{2}-1} \sqrt{1-y^{2}} \sin \zeta
\end{aligned}
$$

The constant $\lambda$ determines the ellipticity of the coordinates.

The inverse coordinate transformation is given by

$$
\begin{aligned}
& x(\rho, z, \varphi)=\frac{1}{2 \lambda}\left[\sqrt{\rho^{2}+\varphi^{2}+(z+\lambda)^{2}}+\sqrt{\rho^{2}+\varphi^{2}+(z-\lambda)^{2}}\right], \\
& y(\rho, z, \varphi)=\frac{1}{2 \lambda}\left[\sqrt{\rho^{2}+\varphi^{2}+(z+\lambda)^{2}}-\sqrt{\rho^{2}+\varphi^{2}+(z-\lambda)^{2}}\right], \\
& \zeta(\rho, z, \varphi)=\arctan \left(\frac{\varphi}{\rho}\right) .
\end{aligned}
$$

In this set of coordinates the axisymmetry simplification become $\zeta=0$, giving exactly eq. (5.27). In case we want to pass from these to the radial coordinates centred in one of the two sources, (5.27) have to be combined with (5.35)-(5.36), as follows

$$
\left\{\begin{array}{l}
x=\frac{1}{2 \lambda}\left[\bar{r}+\sqrt{\bar{r}^{2}-4 \lambda \bar{r} \cos \theta+4 \lambda^{2}}\right], \\
y=\frac{1}{2 \lambda}\left[\bar{r}-\sqrt{\bar{r}^{2}-4 \lambda \bar{r} \cos \theta+4 \lambda^{2}}\right] .
\end{array}\right.
$$

Finally the three-dimesional gradient and laplacian in coordinares (A.8)-(A.10) reads ${ }^{28}$

$$
\begin{aligned}
\vec{\nabla} f(x, y, \zeta) & =\frac{\vec{e}_{x}}{\lambda} \sqrt{\frac{x^{2}-1}{x^{2}-y^{2}}} \frac{\partial f}{\partial x}+\frac{\vec{e}_{y}}{\lambda} \sqrt{\frac{1-y^{2}}{x^{2}-y^{2}}} \frac{\partial f}{\partial y}+\frac{\vec{e}_{y}}{\lambda \sqrt{x^{2}-1} \sqrt{1-y^{2}}} \frac{\partial f}{\partial \zeta}, \\
\nabla^{2} f(x, y, \zeta) & =\frac{1}{\lambda^{2}\left(x^{2}-y^{2}\right)}\left\{\frac{\partial}{\partial x}\left[\left(x^{2}-1\right) \frac{\partial f}{\partial x}\right]+\frac{\partial}{\partial y}\left[\left(1-y^{2}\right) \frac{\partial f}{\partial y}\right]\right\}+\frac{1}{\lambda^{2}\left(x^{2}-1\right)\left(1-y^{2}\right)} \frac{\partial^{2} f}{\partial \zeta^{2}}
\end{aligned}
$$

\section{B Kerr-Newman-NUT solution from the enhanced Ehlers transfor- mation}

For sake of completeness we sum up the main results of the generalised enhanced EhlersReina-Treves transformation applied to the Kerr-Newman black hole to obtain the KerrNewman-NUT solution.

In practice we apply, instead of the standard Ehlers transformation (2.13)-(III) the enhanced generalised Reina-Treves transforamtion (4.9), to the Kerr-Newman seed $\left(\mathcal{E}_{0}, \boldsymbol{\Phi}_{0}\right)$

\footnotetext{
${ }^{28}$ In the reference [42] and in the Carmeli book there are some differences in the form of the differential operators, probably due to typos.
} 
of eqs. (3.12)-(3.11). Thus instead of (3.13)-(3.14) we have

$$
\begin{aligned}
\mathcal{E}_{0}(r, x) \longrightarrow \mathcal{E}_{N}(r, x) & =\frac{\mathcal{E}_{0}(r, x)+i b}{1+i b \mathcal{E}_{0}(r, x)}=1+\frac{2 m(i+b)}{a x-i r+b(r-2 m+i a x)}, \\
\boldsymbol{\Phi}_{0}(r, x) \longrightarrow \boldsymbol{\Phi}(r, x) & =\frac{\boldsymbol{\Phi}_{0}(r, x)(1+i b)}{1+i b \mathcal{E}_{0}(r, x)}=\frac{(b-i)(q+i p)}{a x-i r+b(r-2 m+i a x)},
\end{aligned}
$$

thus, thanks to the definitions (2.9), (2.10) and (2.11), we obtain

$$
\begin{aligned}
& f(r, x)=\operatorname{Re}\left(\mathcal{E}_{N}\right)+\boldsymbol{\Phi}_{N} \boldsymbol{\Phi}_{N}^{*}=\frac{\left(1+b^{2}\right)\left(p^{2}+q^{2}-2 m r+r^{2}+a^{2} x^{2}\right)}{r^{2}-4 a b m x+a^{2} x^{2}+b^{2}\left[\left(r^{2}-2 m\right)^{2}+a^{2} x^{2}\right]} \\
& A_{t}(r, x)=\operatorname{Re}\left(\boldsymbol{\Phi}_{N}\right)=\frac{2 b m p-q r-a p x+b^{2}(2 m q-q r-a p x)}{r^{2}-4 a b m x+a^{2} x^{2}+b^{2}\left[\left(r^{2}-2 m\right)^{2}+a^{2} x^{2}\right]} \\
& \omega(r, x)=-\frac{4 b m x}{1+b^{2}}-\frac{a\left(1-x^{2}\right)\left\{p^{2}+q^{2}-2 m r+b^{2}\left[p^{2}+q^{2}+2 m(r-2 m)+4 a b m x\right]\right\}}{\left(r^{2}-2 m r+q^{2}+p^{2}+a^{2} x^{2}\right)\left(1+b^{2}\right)}+\omega_{0}, \\
& A_{\varphi}(r, x)=A_{\varphi 0}+p x-\left[\frac{4 m x b}{1+b^{2}}+a\left(1-x^{2}\right)-\omega_{0}\right] A_{t}(r, x) .
\end{aligned}
$$

Then the standard Kerr-Newman solution (3.22), (3.31)-(3.37) can be obtained by the following mass and radial coordinate shift:

$$
m \longrightarrow \bar{m}=\sqrt{m^{2}-\ell^{2}}, \quad r \longrightarrow \bar{r}=r+\bar{m}-\sqrt{\bar{m}^{2}+\ell^{2}} .
$$

In this case $t, a, q, p$ remain the same. The nut parameter $\ell$ is related to the enhanced generalised Reina-Treves transformation parameter $b$ through the equation

$$
b=\frac{-\bar{m}+\sqrt{\bar{m}^{2}+\ell^{2}}}{\ell} .
$$

As can be easily understood the new transformation is more economical because no extra transformation is required and minimal adjustment of the parameter and coordinates is needed. Moreover it shorten the results, therefore it simplifies also the interpretation of the generated output, in particular when the output is a novel unknown solution.

In order to support the role of the enhanced Ehlers transformation as the gravitationalgravitomagnetic duality, as proposed in section 4 , it is worth also to write explicitly the falloff of the Ernst complex field of the Kerr-Newman solution after the enhanced Ehlers transformation (4.9)

$$
\begin{aligned}
\mathcal{E}(r, x) & \sim 1-\frac{2 m}{r} \frac{i+b}{i-b}-\frac{2 m(i+b)(a x-2 b m+i a b x)}{(1-b)^{2} r^{2}}+O\left(\frac{1}{\bar{r}^{3}}\right), \\
\mathbf{\Phi}(r, x) & \sim \frac{-q-i p}{\bar{r}}+\frac{(q+i p)(2 b m-a x-i a b x)}{(i-b) r^{2}}+O\left(\frac{1}{\bar{r}^{3}}\right) .
\end{aligned}
$$

We can understand from eq. (B.9) that, if the seed spacetime is not endowed with mass charge, the (enanched) Ehlers transformation is not adding any nut charge, as noted in 
section 4. This occurs because the role of (enanched) Ehlers transformation is just to rotate the gravitational mass charge into the gravomagnetic charge, in the same way the electromagnetic duality rotates the electric monopole charge into the magnetic charge (or viceversa), but the latter is not generating any magnetic charge from electric uncharged seed. A further advantage of the enhanced Ehlers transformation, with respect to the traditional one, is revealed by this duality. Indeed for the standard Ehlers transformation the gravitational duality is not clear because it mixes also the electric with the magnetic field and because non-trivial coordinate transformations are needed to obtain the desidered spacetime.

Thanks to the parameter rescaling ${ }^{29}$ (B.7) we get the standard (dyonic) Kerr-NewmanNUT asymptotic for the complex fields

$$
\begin{aligned}
\mathcal{E}(\bar{r}, x) & \sim 1-2 \frac{\bar{m}-i \ell}{\bar{r}}-\frac{2(\bar{m}-i \ell)\left(-i a x+i \ell-2 \bar{m}+\sqrt{m^{2}+\ell^{2}}\right)}{\bar{r}^{2}}+O\left(\frac{1}{\bar{r}^{3}}\right), \\
\boldsymbol{\Phi}(\bar{r}, x) & \sim \frac{-q-i p}{\bar{r}}+\frac{(p-i q)\left(-a x+\ell+2 i \bar{m}-i \sqrt{\bar{m}^{2}+\ell^{2}}\right)}{\bar{r}^{2}}+O\left(\frac{1}{\bar{r}^{3}}\right),
\end{aligned}
$$

From the latter expansion it is straightforward to identify the conserved quantities of the solution: the mass $\bar{m}$, nut charge $\ell$, angular momentum $J=a m$, electric and magnetic charges $-q$ and $-p$ respectively, electric and magnetic dipole $D_{e}=-a p$ and $D_{q}=a q$.

Note that the complete rotation of the mass charge into the gravitomagnetic one occurs for $b=1$. In the example considered in this appendix it means $\bar{m}=0$ or $m=\ell$.

\section{B.1 Multipole moments expansion for the Kerr-NUT spacetime}

It is clarifying to analyse the mass and the angular multipolar expansion for a metric possessing both angular momentum and the NUT parameter in order to understand the contribution of the NUT parameter to the rotation of the spacetime. Probably the easiest example is just given by the above Kerr-NUT metric, so we can consider the electromagnetic charges $q$ and $p$ null in this subsection.

It is possible to compute the multipoles expansion directly from the Ernst potential (B.1), as explained in [58]. The first four orders of the mass and angular multipoles are respectively given by the following formulas

$$
\begin{aligned}
M_{l} & =\operatorname{Re}\left[\left.\frac{1}{l !} \frac{d \tilde{\xi}(\tilde{z}, 1)}{d \tilde{z}}\right|_{\tilde{z}=0}\right], \\
J_{l} & =\operatorname{Im}\left[\left.\frac{1}{l !} \frac{d \tilde{\xi}(\tilde{z}, 1)}{d \tilde{z}}\right|_{\tilde{z}=0}\right],
\end{aligned}
$$

where $\tilde{\xi}(\tilde{z}, 1)=\frac{1}{\tilde{z}} \xi(\tilde{z}, 1)$ can be inferred, after a coordinate transformation $r \rightarrow \frac{1}{\tilde{z}}+M$ which locates the spacelike infinity at $\tilde{z}=0$, from the gravitational Ernst potential (B.1)

\footnotetext{
${ }^{29}$ The radial shift in (B.7) is not relevant, it is just changing the geometrical constant $z *$ of the Ernst potential expansion (4.11)-(4.12), which is related to the set of coordinate under consideration.
} 
evaluated on the azimuthal symmetry axis $x=1$

$$
\xi(\tilde{z}, 1)=\frac{1-\mathcal{E}_{N}(\tilde{z}, 1)}{1+\mathcal{E}_{N}(\tilde{z}, 1)}
$$

Let us write explicitly, once we have defined $\sigma:=\sqrt{M^{2}+\ell^{2}}$, the first four orders for the Kerr-NUT black hole:

$$
\begin{aligned}
M_{0} & =M, \\
M_{1} & =-M^{2}-a \ell+M \sigma, \\
M_{2} & =-a^{2} M+2 a \ell(M-\sigma)+M\left[\ell^{2}+2 M(M-\sigma)\right], \\
M_{3} & =a^{3} \ell+3 a^{2} M(M-\sigma)+M\left(-4 M^{3}-3 M \ell^{2}+4 M^{2} \sigma+\ell^{2} \sigma\right)-3 a \ell\left[\ell^{2}+2 M(M-\sigma) \sigma\right], \\
J_{0} & =\ell, \\
J_{1} & =-a M+\ell(M-\sigma), \\
J_{2} & =(a-\ell)[\ell(a+\ell)+2 M(M-\sigma)], \\
J_{3} & =a^{3} M+4 M^{3} \ell+3 M \ell^{3}-4 M^{2} \ell \sigma-\ell^{3} \sigma+3 a^{2} \ell(\sigma-M)-3 a M\left[\ell^{2}+2 M(M-\sigma)\right] .
\end{aligned}
$$

When the NUT parameter vanish, i.e. $\ell \rightarrow 0$, these values simplify to the Kerr multipole moment expansion

$$
\begin{aligned}
& M_{0}=M, \quad M_{1}=0, \quad M_{2}=-a^{2} M, \quad M_{3}=0, \\
& J_{0}=0, \quad J_{1}=-a M, \quad J_{2}=0, \quad J_{3}=a^{3} M .
\end{aligned}
$$

From the above example we can see as the NUT parameter $\ell$ contribute to the multipole moments, in particular it represent the angular monopole moment of the source. It is clear that also in the case of null Kerr rotational parameter $a$, the resulting Taub-NUT spacetime is not static but is stationary rotating, even though its angular momentum (proportional to the angular dipole moment $J_{1}$ ) is null, as can be seen from the $a \rightarrow 0$ limit of the expansion (B.16)

$$
\begin{aligned}
M_{0} & =M, \\
M_{1} & =-M^{2}+M \sigma, \\
M_{2} & =M\left[\ell^{2}+2 M(M-\sigma)\right], \\
M_{3} & =M\left(-4 M^{3}-3 M \ell^{2}+4 M^{2} \sigma+\ell^{2} \sigma\right), \\
J_{0} & =\ell, \\
J_{1} & =\ell(M-\sigma), \\
J_{2} & =-\ell\left[\ell^{2}+2 M(M-\sigma)\right], \\
J_{3} & =4 M^{3} \ell+3 M \ell^{3}-4 M^{2} \ell \sigma-\ell^{3} \sigma .
\end{aligned}
$$

Moreover the NUT parameter $\ell$ switches on all the even multipole moments which otherwise would have been null.

For the double Reissner-Nordstrom-NUT spacetime, both in the extremal and nonextremal case, build in section 5 the NUT parameter has a similar behaviour as can be read 
directly from the asymptotic expansion of the complex Ernst potentials (5.17)-(5.18), which capture the first orders of the multipole moments, as already commented in section 5.1. Therefore the double Reissner-Nordstrom-NUT spacetime posses a kind of stationary rotation even though its angular momentum is null, which, similarly to the standard angular momentum, is able to twist geodesics.

\section{NUT generalisation of the Majumdar-Papapetrou in $(\rho, z)$ coordi- nates}

It is not difficult to get the NUT generalisation of the Majumdar-Papapetrou metric in the original cylindrical coordinates $(\rho, z)$ of the Lewis-Wayl-Papapetrou metric (2.4)

$$
d s^{2}=-f(d t+\omega d \varphi)^{2}+f^{-1}\left[\rho^{2} d \varphi^{2}+e^{2 \gamma}\left(d \rho^{2}+d z^{2}\right)\right] .
$$

One has just to transform the functions given in (5.30)-(5.33) according with the coordinates transformation (5.27) to get

$$
\begin{aligned}
f^{-1}(\rho, \theta) & =\left[\frac{1-b^{2}}{1+b^{2}}+\frac{m_{1}}{\sqrt{\rho^{2}+(z+\lambda)^{2}}}+\frac{m_{2}}{\sqrt{\rho^{2}+(z-\lambda)^{2}}}\right]^{2}+\left(\frac{2 b}{1+b^{2}}\right)^{2}, \\
\omega(\rho, \theta) & =\frac{4 b}{1+b^{2}}\left[\frac{m_{1}(z+\lambda)}{\sqrt{\rho^{2}+(z+\lambda)^{2}}}+\frac{m_{2}(z-\lambda)}{\sqrt{\rho^{2}+(z-\lambda)^{2}}}\right]+\omega_{0}, \\
\gamma(\rho, \theta) & =0, \\
A_{t}(\rho, \theta) & =f(\rho, \theta)\left\{\left(\frac{1-b^{2}}{1+b^{2}}\right)\left[\frac{1}{2}\left(\frac{1+b^{2}}{1-b^{2}}\right)+\frac{m_{1}}{\sqrt{\rho^{2}+(z+\lambda)^{2}}}+\frac{m_{2}}{\sqrt{\rho^{2}+(z-\lambda)^{2}}}\right]^{2}-\frac{1}{4}\left(\frac{1+b^{2}}{1-b^{2}}\right)\right\} \\
A_{\varphi}(\rho, \theta) & =\omega(\rho, \theta) A_{t}(\rho, \theta)+A_{\varphi_{0}} .
\end{aligned}
$$

In this form the solution is not only more compact, but also it is more suitable to describe the near horizon geometry of the two sources simultaneously, as done in (5.69).

Open Access. This article is distributed under the terms of the Creative Commons Attribution License (CC-BY 4.0), which permits any use, distribution and reproduction in any medium, provided the original author(s) and source are credited.

\section{References}

[1] W. Israel and K.A. Khan, Collinear particles and bondi dipoles in general relativity, Nuovo Cim. 33 (1964) 331.

[2] W.B. Bonnor, An exact solution of the Einstein-Maxwell equations referring to a magnetic dipole, Z. Phy. 190 (1966) 444.

[3] P.T. Chrusciel, H.S. Reall and P. Tod, On Israel-Wilson-Perjes black holes, Class. Quant. Grav. 23 (2006) 2519 [gr-qc/0512116] [INSPIRE].

[4] D. Kramer and G. Neugebauer, The superposition of two Kerr solutions, Phys. Lett. A 75 (1980) 259 
[5] W. Dietz and C. Hoenselaers, Two mass solutions of Einstein's vacuum equations: The double Kerr solution, Ann. Phys. 165 (1985) 319.

[6] J. Hennig, On the balance problem for two rotating and charged black holes, Class. Quant. Grav. 36 (2019) 235001 [arXiv: 1906.04847] [INSPIRE].

[7] B. Carter, Killing horizons and orthogonally transitive groups in space-time, J. Math. Phys. 10 (1969) 70 [INSPIRE].

[8] F.J. Ernst, New Formulation of the Axially Symmetric Gravitational Field Problem. II, Phys. Rev. 168 (1968) 1415 [INSPIRE].

[9] F. Ernst, Removal of the nodal singularity of the C-metric, J. Math. Phys. 17 (1976) 515.

[10] J.B. Griffiths and J. Podolsky, A New look at the Plebanski-Demianski family of solutions, Int. J. Mod. Phys. D 15 (2006) 335 [gr-qc/0511091] [inSPIRE].

[11] I. Cabrera-Munguia, Unequal binary configurations of interacting Kerr black holes, Phys. Lett. B 786 (2018) 466 [arXiv: 1806.05442] [INSPIRE].

[12] V.S. Manko and E. Ruiz, Metric for two arbitrary Kerr sources, Phys. Lett. B 794 (2019) 36 [arXiv: 1806.10408] [INSPIRE].

[13] W. Kinnersley and D.M. Chitre, Symmetries of the stationary Einstein-Maxwell equations. IV. Transformations which preserve asymptotic flatness, J. Math. Phys. 19 (1978) 2037.

[14] M. Astorino, Pair Creation of Rotating Black Holes, Phys. Rev. D 89 (2014) 044022 [arXiv: 1312.1723] [INSPIRE].

[15] G.A. Alekseev and V.A. Belinski, Superposition of fields of two Reissner-Nordstrom sources, in proceedings of the 11th Marcel Grossmann Meeting on General Relativity, Berlin, Germany, 23-29 July 2006, pp. 2490-2492 [arXiv:0710.2515] [INSPIRE].

[16] G.A. Alekseev and V.A. Belinski, Equilibrium configurations of two charged masses in General Relativity, Phys. Rev. D 76 (2007) 021501 [arXiv:0706.1981] [InSPIRE].

[17] V.S. Manko, The Double-Reissner-Nordstrom solution and the interaction force between two spherically symmetric charged particles, Phys. Rev. D 76 (2007) 124032 [arXiv:0710.2158] [INSPIRE].

[18] I. Cabrera-Munguia, V.S. Manko and E. Ruiz, A combined Majumdar-Papapetrou-Bonnor field as extreme limit of the double-Reissner-Nordstrom solution, Gen. Rel. Grav. 43 (2011) 1593 [arXiv: 0911.0756] [INSPIRE].

[19] G. Neugebauer and J. Hennig, Stationary two-black-hole configurations: A non-existence proof, J. Geom. Phys. 62 (2012) 613 [arXiv:1105.5830] [InSPIRE].

[20] G. Bossard, H. Nicolai and K.S. Stelle, Gravitational multi-NUT solitons, Komar masses and charges, Gen. Rel. Grav. 41 (2009) 1367 [arXiv: 0809.5218] [INSPIRE].

[21] S.D. Majumdar, A class of exact solutions of Einstein's field equations, Phys. Rev. 72 (1947) 390 [INSPIRE].

[22] A. Papapetrou, A static solution of the equations of the gravitational field for an arbitary charge-distribution, Proc. Roy. Irish Acad. A 51 (1947) 191 and online at https://www.jstor.org/stable/20488481.

[23] W. Israel and G.A. Wilson, A class of stationary electromagnetic vacuum fields, J. Math. Phys. 13 (1972) 865 [INSPIRE]. 
[24] Z. Perjés, Solutions of the coupled Einstein Maxwell equations representing the fields of spinning sources, Phys. Rev. Lett. 27 (1971) 1668 [INSPIRE].

[25] J.B. Hartle and S.W. Hawking, Solutions of the Einstein-Maxwell equations with many black holes, Commun. Math. Phys. 26 (1972) 87 [InSPIRE].

[26] S. Chandrasekhar, The Mathematical Theory of Black Holes, Oxford University Press, Oxford U.K. (1998).

[27] F.J. Ernst, Black holes in a magnetic universe, J. Math. Phys. 17 (1976) 54 [InSPIRE].

[28] G.A. Alekseev and V.A. Belinski, Superposition of fields of two rotating charged masses in general relativity and existence of equilibrium configurations, Gen. Rel. Grav. 51 (2019) 68 [arXiv: 1905.05317] [INSPIRE].

[29] G. Clément, D. Gal'tsov and M. Guenouche, Rehabilitating space-times with NUTs, Phys. Lett. B 750 (2015) 591 [arXiv: 1508. 07622] [INSPIRE].

[30] G. Clément, D. Gal'tsov and M. Guenouche, NUT wormholes, Phys. Rev. D 93 (2016) 024048 [arXiv: 1509.07854] [INSPIRE].

[31] M. Guica, T. Hartman, W. Song and A. Strominger, The Kerr/CFT Correspondence, Phys. Rev. D 80 (2009) 124008 [arXiv:0809.4266] [INSPIRE].

[32] T. Hartman, K. Murata, T. Nishioka and A. Strominger, CFT Duals for Extreme Black Holes, JHEP 04 (2009) 019 [arXiv:0811.4393] [InSPIRE].

[33] I. Bredberg, C. Keeler, V. Lysov and A. Strominger, Cargese Lectures on the Kerr/CFT Correspondence, Nucl. Phys. Proc. Suppl. 216 (2011) 194 [arXiv:1103.2355] [InSPIRE].

[34] G. Compère, The Kerr/CFT correspondence and its extensions, Living Rev. Rel. 15 (2012) 11 [Living Rev. Rel. 20 (2017) 1] (revised version) [arXiv:1203.3561] [INSPIRE].

[35] D.R. Brill, Electromagnetic Fields in a Homogeneous, Nonisotropic Universe, Phys. Rev. 133 (1964) B845.

[36] M. Astorino, Microscopic Entropy of the Magnetised Extremal Reissner-Nordstrom Black Hole, JHEP 10 (2015) 016 [arXiv: 1507.04347] [INSPIRE].

[37] M. Astorino, CFT Duals for Accelerating Black Holes, Phys. Lett. B 760 (2016) 393 [arXiv: 1605.06131] [INSPIRE].

[38] M.F.A.R. Sakti, A. Suroso and F.P. Zen, CFT duals on extremal rotating NUT black holes, Int. J. Mod. Phys. D 27 (2018) 1850109 [arXiv: 1712.04751] [InSPIRE].

[39] H.K. Kunduri, J. Lucietti and H.S. Reall, Near-horizon symmetries of extremal black holes, Class. Quant. Grav. 24 (2007) 4169 [arXiv:0705.4214] [InSPIRE].

[40] H. Stephani, D. Kramer, M.A.H. MacCallum, C. Hoenselaers and E. Herlt, Exact solutions of Einstein's field equations, Cambridge University Press, Cambridge U.K. (2009) [INSPIRE].

[41] M. Astorino, Charging axisymmetric space-times with cosmological constant, JHEP 06 (2012) 086 [arXiv:1205.6998] [inSPIRE].

[42] A. Reina and A. Treves, NUT-like generalization of axisymmetric gravitational fields, J. Math. Phys. 16 (1975) 834.

[43] Y.-T. Huang, U. Kol and D. O'Connell, The Double Copy of Electric-Magnetic Duality, arXiv:1911.06318 [INSPIRE]. 
[44] J.F. Plebanski and M. Demianski, Rotating, charged and uniformly accelerating mass in general relativity, Annals Phys. 98 (1976) 98 [INSPIRE].

[45] B. Carter, Mathematical foundations of the theory of relativistic stellar and black hole configurations, Gravitation in Astrophysics. NATO ASI Series, volume 156, Springer, Boston MA U.S.A. (1987), pp. 63-122.

[46] D. Garfinkle and R.B. Mann, Generalized entropy and Noether charge, Class. Quant. Grav. 17 (2000) 3317 [gr-qc/0004056] [INSPIRE].

[47] C. Teitelboim, Action and entropy of extreme and nonextreme black holes, Phys. Rev. D 51 (1995) 4315 [Erratum ibid. D 52 (1995) 6201] [hep-th/9410103] [InSPIRE].

[48] S.W. Hawking, G.T. Horowitz and S.F. Ross, Entropy, Area and black hole pairs, Phys. Rev. D 51 (1995) 4302 [gr-qc/9409013] [INSPIRE].

[49] G.W. Gibbons and R.E. Kallosh, Topology, entropy and Witten index of dilaton black holes, Phys. Rev. D 51 (1995) 2839 [hep-th/9407118] [INSPIRE].

[50] A. Strominger and C. Vafa, Microscopic origin of the Bekenstein-Hawking entropy, Phys. Lett. B 379 (1996) 99 [hep-th/9601029] [INSPIRE].

[51] P. Mitra, Entropy of extremal black holes, in proceedings of the Workshop on Frontiers in Field Theory, Quantum Gravity and String Theory, Puri, India, 12-21 December 1996, pp. 255-263 [hep-th/9704201] [INSPIRE].

[52] S.M. Carroll, M.C. Johnson and L. Randall, Extremal limits and black hole entropy, JHEP 11 (2009) 109 [arXiv:0901.0931] [INSPIRE].

[53] R.A. Hennigar, D. Kubizňák and R.B. Mann, Thermodynamics of Lorentzian Taub-NUT spacetimes, Phys. Rev. D 100 (2019) 064055 [arXiv: 1903.08668] [INSPIRE].

[54] J. Ciafre and M.J. Rodriguez, A Near Horizon Extreme Binary Black Hole Geometry, Eur. Phys. J. C 79 (2019) 754 [arXiv: 1804.06985] [INSPIRE].

[55] M. Astorino, Embedding hairy black holes in a magnetic universe, Phys. Rev. D 87 (2013) 084029 [arXiv: 1301.6794$]$ [INSPIRE].

[56] M. Astorino, Stationary axisymmetric spacetimes with a conformally coupled scalar field, Phys. Rev. D 91 (2015) 064066 [arXiv:1412.3539] [INSPIRE].

[57] Y. Bardoux, M.M. Caldarelli and C. Charmousis, Integrability in conformally coupled gravity: Taub-NUT spacetimes and rotating black holes, JHEP 05 (2014) 039 [arXiv:1311.1192] [INSPIRE].

[58] H. Quevedo, Multipole Moments in General Relativity - Static and Stationary Vacuum Solutions, Fortsch. Phys. 38 (1990) 733. 